\title{
Lapurdum
}

Euskal ikerketen aldizkaria | Revue d'études basques |

Revista de estudios vascos | Basque studies review

$7 \mid 2002$

Numéro VII

\section{Aménagement linguistique et vitalité des communautés francophone et anglophone du Québec}

Richard Y. Bourhis et Dominique Lepicq

\section{OpenEdition}

\section{Journals}

Édition électronique

URL : http://journals.openedition.org/lapurdum/981

DOI : 10.4000/lapurdum.981

ISSN : 1965-0655

Éditeur

IKER

Édition imprimée

Date de publication : 1 octobre 2002

Pagination : 137-176

ISBN : 2-86781-321-2

ISSN : $1273-3830$

Référence électronique

Richard Y. Bourhis et Dominique Lepicq, «Aménagement linguistique et vitalité des communautés francophone et anglophone du Québec », Lapurdum [En ligne], 7 | 2002, mis en ligne le 01 juin 2009, consulté le 19 avril 2019. URL : http://journals.openedition.org/lapurdum/981 ; DOI : 10.4000/ lapurdum.981 


\title{
Aménagement linguistique et vitalité des communautés francophone et anglophone du Québec
}

\author{
Richard Y. BOURHIS \\ Université du Québec à Montréal \\ Dominique LEPICQ ${ }^{2}$ \\ McMaster University, Hamilton
}

Le cas du Québec français montre qu'un aménagement linguistique soutenu en faveur d'une langue minoritaire à l'échelle continentale peut mettre un terme au glissement linguistique vers la langue la plus puissante de ce millénaire : l'anglais. L'objectif de cet article est de fournir un survol de la situation sociolinguistique au Québec suite à l'adoption de lois linguistiques destinées à asseoir le statut du français par rapport à l'anglais dans le seul territoire majoritairement francophone de l'Amérique du Nord. En s'inspirant du concept de vitalité linguistique, la première partie de cet article analyse les circonstances qui ont mené les gouvernements successifs du Québec à adopter des lois linguistiques en faveur du français durant les trois dernières décennies. La seconde partie de l'article fait le bilan de la situation démolinguistique du français et de l'anglais après vingt ans d'aménagement linguistique. Nous conclurons en examinant la façon dont les politiques linguistiques ont influencé la vitalité des communautés francophone et anglophone du Québec.

\section{1) La Vitalité linguistique : le cas du Québec}

Les mouvements culturels de revitalisation qui se manifestèrent à travers le monde dans la dernière partie du $20^{\text {ème }}$ siècle avaient souvent comme revendication principale le maintien et l'épanouissement des langues minoritaires (Fishman, 1999). Le modèle de la vitalité linguistique a été mis au point pour analyser de façon plus systématique dans une optique comparative la force relative des

\footnotetext{
' Richard Y. Bourhis est professeur au département de psychologie à l'Université du Québec à Montréal, directeur à l'UQAM de la Chaire Concordia-UQAM en études ethniques, chercheur au Centre d'études ethniques des universités montréalaises (CEETUM). E-mail bourhis.richard@uqam.ca

2 Dominique Lepicq est professeur au département de français à McMaster University, Hamilton, Ontario, Canada.
} 
communautés linguistiques en contact (Giles, Bourhis et Taylor, 1977). Comme le signalent Harwood, Giles et Bourhis (1994, 172) :

«Il devint évident qu'on ne pouvait plus étudier des processus tels que les transferts linguistiques, les attitudes linguistiques, la communication interculturelle et les conflits linguistiques dans un vacuum sociostructural.... A cet égard, Johnson et al. (1983) ont su montrer que le concept de vitalité résulte du besoin de resituer les processus sociolinguistiques et socio-psychologiques sous-jacents aux comportements intergroupes au sein de leur propre contexte sociostructural».(p.172)

En fait, le modèle de vitalité linguistique fut conçu à l'origine pour le contexte du Québec à une époque où la recherche sociolinguistique québécoise avait pour but d'élaborer des politiques linguistiques destinées à rehausser le statut du français par rapport à l'anglais. (Giles, Bourhis et Taylor, 1977). Ainsi, le concept de vitalité linguistique fut conçu comme un instrument analytique destiné à mieux évaluer la force démographique, le soutien institutionnel et le prestige de la majorité francophone du Québec par rapport à l'élite anglophone de l'époque. La vitalité d'un groupe linguistique se définit comme «ce qui rend un groupe susceptible de fonctionner en tant que collectivité distincte et active au sein d'un contexte intergroupe régional ou national» (Giles et al., 1977 : 308). Plus on estime que la vitalité d'un groupe linguistique est élevée, plus ce groupe a de chance de survivre en tant que collectivité distincte au sein de son contexte bilingue ou multilingue. Inversement, des groupes linguistiques dont la vitalité est jugée faible seraient voués à une disparition éventuelle en tant que communautés linguistiques distinctes. La figure 1 montre les trois principales dimensions des variables structurales susceptibles d'influencer la vitalité des groupes linguistiques, à savoir la force démographique, le contrôle et le soutien institutionnel et les variables de statut. Nous donnerons ci-dessous une brève description des éléments qui composent ces facteurs dans la mesure où ils caractérisent la situation des communautés anglophone et francophone du Québec durant la décennie menant à l'adoption de la loi 101 .

_ (voir fig. 1)

Les variables démographiques ont trait au nombre de membres composant le groupe linguistique et à leur densité à l'intérieur d'un territoire urbain, régional ou national. Les facteurs de densité réfèrent à la concentration numérique des membres de l'endogroupe linguistique dans différentes zones du territoire, à leur proportion par rapport aux membres de l'exogroupe et au fait que l'endogroupe occupe encore ou non son territoire «ancestral» ou «national». Les variables démographiques correspondent aussi au nombre total des membres de la communauté linguistique en termes absolus, son taux de fécondité et de mortalité, son degré d'endogamie ou d'exogamie et ses tendances à l'immigration et à l'émigration.

Sans nécessairement utiliser le modèle de vitalité en tant que tel, les chercheurs canadiens en sciences sociales ont pu établir un tableau fidèle de la vitalité démographique des francophones et des anglophones du Canada au cours des années 1970. Pour la plupart, ces recherches ont été effectuées dans le cadre de deux enquêtes gouvemementales de grande envergure, l'une sous l'égide du gouvernement fédéral examinant les relations français-anglais à l'échelle du Canada (Commission royale sur le bilinguisme et le biculturalisme, Rapport B\&B ; Canada, 
1969), l'autre sous l'égide du gouvernement du Québec, la "Commission Gendron» axée sur l'évolution sociolinguistique du français dans la province (Québec, 1972).

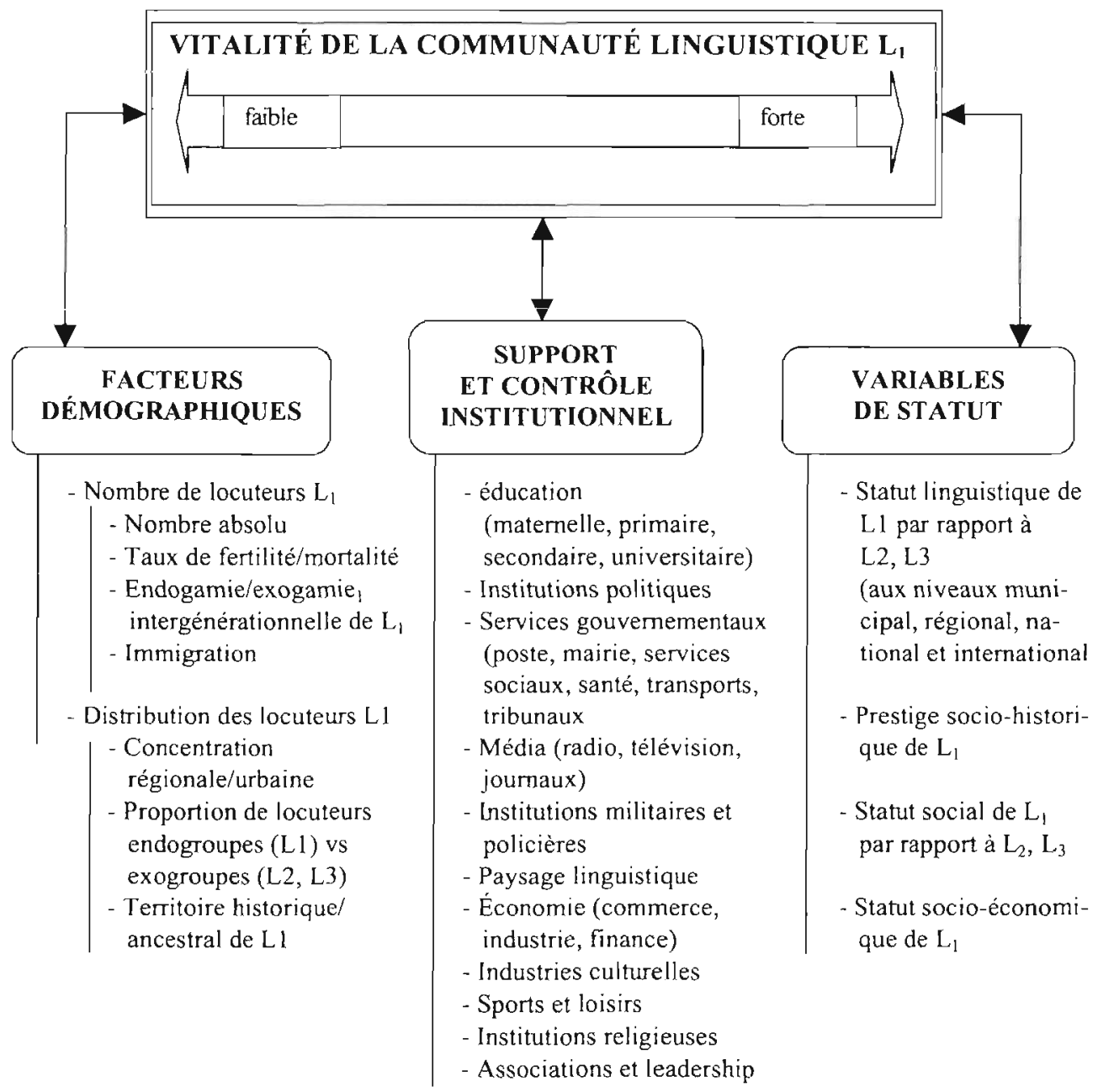

Figure 1 - Taxonomie des facteurs socio-structuraux qui affectent la vitalité d'une communauté linguistique $L_{1}$ en contact avec des communautés linguistiques exogroupes $L_{2}, L_{3}$ sur un même territoire (adapté de Bourhis, 2001)

Les analyses démolinguistiques fournies dans le rapport $B \& B$ (Canada, 1969) ont confirmé la perception des Québécois selon laquelle les francophones hors Québec étaient soumis à une assimilation soutenue qui risquait de compromettre la 
survie à long terme des minorités francophones dans les provinces anglocanadiennes (Castonguay, 1998; de Vries, 1994). Des facteurs tels que l'isolement des petites communautés francophones à travers le pays, le déclin du taux de natalité, l'exogamie c'est-à-dire les mariages entre francophones et anglophones, l'absence de scolarisation en français au primaire et au secondaire, le legs des lois contre les minorités francophones du Canada et la pénurie de services en français à la fois au niveau provincial et fédéral ont tous contribué à réduire le nombre de francophones hors Québec (Bourhis, 1994a). Ainsi, les études sociolinguistiques des années 1960 soulignaient le déclin des communautés francophones hors Québec, glissement linguistique qui s'effectuait systématiquement au profit de l'anglais, langue dominante du continent. Pour contrer l'assimilation des minorités francophones, le gouvernement fédéral canadien adopta en 1969 la loi sur les langues officielles (Fortier, 1994). Mais, pour bien des francophones du Québec, l'adoption de cette loi garantissant des services en français ou en anglais au niveau fédéral «là où le nombre le justifie» fut considérée comme insuffisante et trop tardive. Forts de ces considérations, les nationalistes québécois adoptèrent le slogan «hors du Québec point de salut». On vit alors resurgir les vieux spectres et les menaces sur la disparition progressive des Canadiens français, non seulement à l'échelle du Canada mais aussi en Amérique du Nord. En tant que minorité linguistique au sein du Canada $(28 \%$ en 1961) et en Amérique du Nord dans son ensemble (moins de $2 \%$ ), le Québec représentait aux yeux de certains «la demière enclave territoriale» dans laquelle une société francophone normale pouvait survivre et même s'épanouir au sein du nouveau monde.

Sur le plan démolinguistique durant la même période, plus de $80 \%$ de la population du Québec parlait le français langue maternelle, dont les trois quarts étaient des francophones unilingues qui utilisaient seulement le français comme langue du foyer. Etant donné le fort taux de transmission intergénérationnelle du français langue maternelle, les politiques linguistiques en faveur du français se faisaient dans des conditions très favorables sociolinguistiquement. En termes de distribution, les francophones constituaient la majorité dans toutes les régions de la province sauf dans certains quartiers de l'île de Montréal. Dans plusieurs quartiers à l'ouest de Montréal, les anglophones, pour la plupart unilingues, étaient majoritaires. Les francophones, quant à eux, étaient majoritaires à l'est et au nord de Montréal. Les anglophones et les francophones étaient répartis de façon plus égale dans les quartiers au centre de Montréal ainsi que dans les régions à l'ouest de la province, voisines de la frontière de l'Ontario et les cantons de l'Est. Les Québécois ayant l'anglais pour langue maternelle formaient moins de $15 \%$ de l'ensemble de la population de la province. Néanmoins, la transmission intergénérationnelle de l'anglais était garantie au sein de la minorité anglophone, soutenue d'ailleurs par les mariages exogames et par le transfert linguistique de beaucoup d'immigrants et d'allophones qui adoptaient l'anglais comme langue du foyer. On utilise le terme allophone pour décrire des individus dont la langue maternelle n'est ni le français ni l'anglais, qu'il s'agisse d'immigrants de première ou de seconde génération. Au début des années 1970, les allophones et les immigrants représentaient $8 \%$ de la population du Québec, la plupart étant établis dans la région de Montréal (88\%) et tendant à adopter l'anglais comme langue d'intégration à la communauté d'accueil anglophone. 
À la même époque, les résultats de la Commission Gendron révélaient des tendances qui étaient moins favorables à la pérennité du fait français au Québec (Québec, 1972). Les démographes confirmaient le déclin de la fécondité des francophones qui, de l'un des plus élevés, était devenu un des plus faibles du monde occidental (Lachapelle et Henripin, 1980). Étant donné que ce taux était inférieur au seuil de remplacement de la population qui est de 2.1 enfants par femme, les francophones ne pouvaient plus compter sur la soi-disant «revanche des berceaux» pour maintenir leur avantage démographique à l'intérieur du Québec (Caldwell et Fournier, 1987). En l'absence d'un taux de natalité soutenu chez les francophones, une immigration accrue et l'intégration des immigrants à la communauté francophone plutôt qu'anglophone apparurent comme des stratégies importantes pour endiguer le déclin à long terme de la population francophone de la province (Termote et Gauvreau, 1988). Pour les mêmes raisons, la minorité anglophone, dont le taux de fécondité n'était pas plus élevé que celui de la majorité francophone, comptait sur les immigrants pour consolider la vitalité démographique de sa propre communauté linguistique.

Libres de choisir entre le système éducatif français ou anglais (de la maternelle à l'université), la majorité des immigrants inscrivaient leurs enfants au système scolaire anglophone afin d'optimaliser la mobilité économique et linguistique de leur progéniture à l'échelle du Canada et de l'Amérique du Nord. En outre, le système scolaire catholique de l'époque (à la fois francophone et anglophone au niveau primaire et secondaire) n'acceptait pas les élèves d'origine immigrante dont la religion était autre que catholique. En revanche, le système protestant (majoritairement anglophone) acceptait les élèves de n'importe quelle confession religieuse (d'Anglejan, 1984). Ainsi, au fil des années, une majorité d'élèves immigrants de première et de seconde génération s'intégrèrent à la communauté d'accueil anglophone plutôt qu'à la communauté d'accueil francophone de la province (Mc Andrew, 1996). Pour beaucoup d'activistes francophones, la liberté du choix de la langue d'enseignement surtout au primaire et au secondaire signifiait que la croissance démographique à long terme allait favoriser la communauté anglophone plutôt que francophone. De plus, certains francophones firent remarquer qu'à moins de contrôler son immigration, le gouvernement du Québec ne pourrait pas prétendre promouvoir l'image de la province comme étant un territoire à majorité francophone et qu'il aurait des difficultés à attirer des immigrants des régions francophones du monde.

Au vu de ces constatations, les analystes québécois fournirent des scénarios optimistes, neutres ou pessimistes sur l'avenir démographique de la population francophone de la province. Mus par des options idéologiques divergentes, les fédéralistes francophones favorisaient les prévisions optimistes concernant la pérennité de la majorité francophone du Québec. Les nationalistes québécois, pour leur part, privilégiaient les pronostics alarmistes, entretenant ainsi l'insécurité des francophones à propos de la survie du «fait français» au Québec. Les tendances démolinguistiques fournies par les chercheurs firent l'objet de rapports détaillés dans les médias assortis de débats sur le partage des pouvoirs entre juridiction fédérale (gouvernement canadien) et provinciale (gouvernement du Québec). Ainsi, les questions liées à la vitalité démographique des communautés anglophone, francophone et allophone de la province furent exploitées de façon stratégique par 
les partis politiques adverses endossant des options contrastées quant à l'avenir politique de la province : souverainistes québécois versus fédéralistes canadiens.

$A u$ sein des démocraties, la vitalité démolinguistique représente «la force du nombre» et peut servir d'instrument légal pour doter la majorité (et dans certains cas les minorités) de la complétude institutionnelle (Breton, 1971) requise pour assurer la pérennité des communautés linguistiques partageant le même territoire national. Selon le schéma de la vitalité, le facteur "contrôle institutionnel» renvoie au niveau de contrôle et de soutien dont jouit un groupe linguistique dans les diverses institutions officielles de l'État (Figure 1). Le soutien et le contrôle institutionnel officiel équivalent au niveau atteint par les membres d'un groupe linguistique dans la gestion et la prise de décision dans des secteurs tels que les affaires, l'industrie, les médias, la production culturelle, le sport, les institutions religieuses et dans les grands corps de l'État tels que l'éducation, la santé, les services sociaux, la police, l'administration et la justice. Les groupes linguistiques qui jouissent d'un contrôle institutionnel élevé au sein de l'État et des institutions privées sont en mesure de sauvegarder et de consolider leur vitalité en tant que collectivité distincte comparativement aux groupes linguistiques qui ne disposent pas d'un tel contrôle sur les institutions. Le soutien institutionnel «non officiel» réfère au niveau d'organisation d'un groupe linguistique constitué en associations pour représenter et sauvegarder ses intérêts linguistiques dans diveries instances privées, par exemple l'enseignement primaire et secondaire privé, les commerces, le sport, la culture et la religion.

Autre facteur qui contribue au contrôle institutionnel des groupes linguistiques : la présence et l'envergure des leaders à la tête des institutions officielles et nonofficielles qui représentent les intérêts de leur endogroupe linguistique. Les progrès liés au contrôle institutionnel dépendent de la présence d'activistes et de leaders charismatiques qui réussissent à mobiliser les membres de leur endogroupe linguistique en les incitant à lutter pour la survie de leur langue et de leur culture en contexte multilingue. L'absence d'un tel leadership, surtout au sein de la minorité linguistique, peut saper les acquis des générations antérieures quant au contrôle institutionnel officiel et non-officiel et peut compromettre les progrès nécessaires à la survie des générations montantes du groupe linguistique en question.

Dès le début des années 1970, à la fois les nationalistes québécois et canadiens se sont distingués par un leadership politique exceptionnel ; parmi eux, citons René Lévesque du côté des souverainistes québécois et Pierre-Elliot Trudeau dans le camp des fédéralistes canadiens. Charismatiques dans leur camp politique respectif, ces deux politiciens rivaux ont incarné l'affrontement des nationalistes québécois et canadiens.

Á l'aube de leur carrière, ces deux leaders ainsi que les citoyens canadiens et québécois furent confrontés aux autres résultats de la Commission royale sur le bilinguisme et le biculturalisme (Canada, 1969). Le rapport montrait non seulement que les francophones étaient sous-représentés dans l'administration fédérale canadienne, l'anglais étant la langue de travail dominante, maís aussi qu'à qualification et expérience égales, le salaire des francophones était systématiquement inférieur à celui des anglophones à travers tout le Canada. Même au Québec, l'écart de salaire était à l'avantage des anglophones unilingues par rapport aux francophones bilingues. Le rapport soulignait que la main mise des Canadiens anglais sur l'économie et les finances contribuait au statut inférieur des 
employés francophones dans les affaires et les entreprises du Québec. Les résultats de la Commission Gendron (Québec, 1972) allaient dans le même sens, à savoir que l'anglais et non le français était la langue de travail et de la mobilité économique au Québec. Vu l'intégration croissante du Québec au sein de l'économie canadienne et américaine, la Commission Gendron concluait que sans l'intervention du gouvernement provincial, la situation diglossique du français irait en s'aggravant face à l'anglais, langue de prestige dans le monde du travail.

Toutefois, sur la plupart des autres facteurs de soutien institutionnel du modèle de vitalité, la situation de la majorité francophone du Québec se présentait sous un jour favorable. Cette situation avantageuse découlait en partie du partage des pouvoirs entre le gouvemement fédéral et les gouvernements provinciaux. En effet, le fédéralisme canadien garantit aux provinces une large part d'autonomie dans des secteurs cruciaux tels que le pouvoir de taxation et de dépenses décidé par les assemblées législatives de chacune des provinces canadiennes. De ce fait, les gouvernements provinciaux contrôlent tous les grands ministères des Etats souverains à l'exception de la Défense nationale, des Affaires étrangères et de la banque centrale. Cet éventail de juridictions provinciales inclut le droit d'offrir des services en français et/ou en anglais dans tous les ministères et services provinciaux. Historiquement, le Québec a su tirer parti de son autonomie provinciale pour assurer à sa majorité francophone le soutien institutionnel nécessaire à son épanouissement linguistique et culturel. Ainsi, les francophones détiennent le contrôle de leurs propres administrations civiles au niveau provincial et municipal ; ils gèrent leur propre système de santé ainsi que leur propre système d'éducation (de la maternelle à l'université). Les francophones sont propriétaires et maîtres de leur propre réseau de médias (radio, télévision, presse, édition) ; ils créent et consomment les produits culturels (musique, littérature, théâtre) et sportifs d'expression française et participent au système politique provincial et fédéral. Bien que traditionnellement privés de la gestion des affaires, de la finance et des banques de leur province, à l'époque, les francophones tiraient une certaine fierté de leur réseau de caisses d'épargne populaires au service des entreprises et commerces endogroupes.

Etant donné son statut historique d'élite dominante, la minorité anglophone a longtemps bénéficié d'un contrôle institutionnel considérable dans la province. A l'époque, non seulement les principales institutions commerciales et financières étaient aux mains des anglophones mais ceux-ci contrôlaient leur propre système de santé (hôpitaux, cliniques) et leur système d'éducation (de la maternelle à l'université) ; ils géraient leur propre réseau confessionnel ainsi que les médias d'expression anglaise (radio ; télévision ; presse). Ils étaient impliqués dans les activités culturelles et sportives au Québec ainsi qu'au Canada et dans le monde anglo-américain. En outre, ils participaient à la politique au niveau municipal, provincial et fédéral.

Les groupes linguistiques qui sont parvenus à un certain niveau de contrôle institutionnel sont par là même susceptibles de jouir d'un statut social supérieur aux groupes moins avantagés démographiquement ou institutionnellement. Les variables de statut sont liées au prestige social d'une communauté linguistique, à son statut socio-historique et au prestige de sa langue et de sa culture à la fois sur le territoire régional, national et sur le plan international (Figure 1). Plus le statut d'une communauté linguistique est perçu comme élevé, plus on lui attribue un haut 
degré de vitalité en tant que collectivité. Bien que le statut d'une communauté linguistique soit plus difficile à quantifier que les variables démographiques et institutionnelles, les données socio-psychologiques montrent que plus un groupe linguistique détient de prestige, plus son identité sociale a des chances d'être positive, ce qui, à son tour, incite les membres à se mobiliser pour accroître la vitalité linguistique de leur endogroupe (Sachdev et Bourhis, 1991). Le fait d'appartenir à un groupe linguistique de faible statut et /ou dévalorisé peut miner la volonté de mobilisation collective nécessaire pour assurer la survie de la langue et de la culture de l'endogroupe. La conscience du statut relatif de l'endogroupe dans la stratification sociale est d'autant plus claire que les différences de statut entre groupes linguistiques se perpétuent à travers les stéréotypes relatifs aux accents, dialectes et langues des locuteurs (Genesee et Bourhis, 1988, Ryan et Giles, 1982), qu'elles s'actualisent dans les normes langagières diglossiques et qu'elles sont officialisées au moyen de lois linguistiques qui reflètent le statut relatif des communautés linguistiques en présence (Wardhaugh, 1987).

L'intérêt durable suscité par «le cas québécois» tient au fait que, dans cette région du monde, l'enjeu réside dans l'affrontement entre deux langues de prestige à la fois sur le plan historique et culturel en occident. Bien que minoritaire en Amérique du Nord, le français jouit d'une plus grande vitalité sur le plan du statut linguistique que s'il s'agissait d'une langue minoritaire de moindre envergure sur le plan historique et culturel. Historiquement, à l'échelle du Québec, la situation diglossique en faveur de l'anglais s'est surtout fait sentir dans le monde du travail dans les zones de contact bilingues à Montréal, dans les régions de l'Ouest le long de la frontière avec l'Ontario et dans les cantons de l'Est le long de la frontière américaine. Une longue tradition de recherche en psychologie sociale sur les attitudes linguistiques et la communication bilingue illustre bien de quelle façon la situation de diglossie a favorisé l'anglais au détriment du français comme langue de prestige dans ces zones de contact (Bourhis, 1994b ; Bourhis et Lepicq, 1993 ; Genesee et Holobow, 1989 ; Hamers et Hummel, 1994 ; Lambert et al., 1960). Des études récentes montrent que des élèves anglophones fréquentant des écoles secondaires de langue anglaise dans des régions majoritairement francophones telles que la ville de Québec sont susceptibles d'utiliser aussi souvent l'anglais dans leur vie quotidienne que de jeunes anglophones vivant dans les quartiers ouest de Montréal où ils constituent la majorité locale (Landry, Allard et Bourhis, 1997). La même étude effectuée auprès d'élèves francophones du secondaire a montré que ceux qui résident dans les quartiers ouest de Montréal majoritairement anglophones sont moins enclins à utiliser le français dans leur vie quotidienne que les jeunes francophones de la ville de Québec, contexte à majorité francophone. Les résultats montrent à la fois la force d'attraction et le prestige de l'anglais qui perdurent chez les francophones et la possibilité pour les Anglo-Québécois de se comporter comme des majoritaires, quelle que soit la densité démographique de leur communauté linguistique à travers la province (Landry et al., 1997).

Giles et al. (1977) ont proposé que les variables démographiques, celles ayant trait au contrôle institutionnel et au statut se combinent pour orienter dans une direction ou une autre la force globale des communautés linguistiques. Tout en étant désavantagé sur le plan démographique, comme c'est le cas des AngloQuébécois, ce même groupe linguistique peut maintenir le contrôle institutionnel sur les médias et posséder un statut exceptionnel grâce au prestige et au 
rayonnement de l'anglais, langue des affaires et des médias à l'échelle de l'Ämérique du Nord. Dans le contexte du Québec des années 1970, on pouvait évaluer la vitalité globale des Anglo-Québécois comme étant moyennement élevée par rapport à des groupes de vitalité faible tels que les immigrants allophones et les minorités autochtones (Premières nations) dispersées dans les réserves à travers la province (Drapeau, 1998 ; Hamers et Hummel, 1998 ; Maurais, 1997). Bien que dotés d'une vitalité élevée sur le plan démographique au Québec, les francophones se sentaient menacés en tant que minorité linguistique sur le continent nordaméricain et avaient une conscience accrue du statut inférieur du français en tant que langue du travail et de la mobilité sociale dans le monde des affaires et de la finance. Des événements politiques ponctuels ainsi qu'une conscience accrue de la précarité du fait français au Québec incita une majorité de francophones à élire le parti québécois pro-indépendantiste en 1976, dont la première décision législative fut l'adoption de la Charte de la langue française (loi 101).

\section{2) Inversion du glissement linguistique des langues en difficulté}

En général, en sociolinguistique, on traduit l'expression anglaise «language transfer» par le terme «transfert linguistique» qui dénote le caractère irréversible de l'attrition d'une langue en difficulté. Entre autres, le transfert linguistique peut signifier le déclin irréversible d'une langue minoritaire au profit d'une langue dominante au fil d'une ou plusieurs générations sur un territoire donné. Dans le modèle de Fishman (1991) «Reversing language shift» (RLS), il convient de parler de «glissement linguistique» puisqu'il s'agit d'un processus qui peut être inversé grâce à l'adoption de politiques linguistiques locales, régionales ou nationales. Ainsi, un aménagement linguistique soutenu en faveur d'une langue minoritaire peut aboutir au «renversement du glissement linguistique» (RGL). Joshua Fishman (1991) a lui-même reconnu la nécessité de tenir compte de la vitalité des minorités linguistiques dans les contextes multilingues complexes :

«A certains égards, le GIDS (Graded Intergenerational Disruption

Scale : Echelle graduée de discontinuité intergénérationnelle d'une langue en difficulté) peut être interprété comme un changement socioculturel analogue aux mesures de vitalité linguistique de nature sociopsychologique proposées récemment par plusieurs chercheurs ; plus le classement sur le GIDS est élevé, moins il y a de continuité intergénérationnelle et donc plus les chances de maintien d'un réseau ou d'une communauté linguistiques sont faibles.» (p. 87)

Le modèle GIDS est basé sur deux critères principaux du glissement linguistique : la transmission intergénérationnelle de la langue maternelle et l'existence d'une norme diglossique réglementant l'usage des langues rivales selon les fonctions de bas ou de haut statut dans la société. Ces deux indices cruciaux du glissement linguistique ne faisaient pas l'objet d'une évaluation particulière dans le modèle original de vitalité linguistique puisque la finalité du modèle n'était pas de servir de guide quant aux modalités de l'aménagement linguistique. Le modèle de vitalité impliquait la diglossie puisqu'il englobait à la fois le soutien institutionnel et le statut linguistique de communautés linguistiques rivales cohabitant sur un 
territoire donné. Le niveau de contrôle institutionnel acquis par une communauté linguistique dans les fonctions officielles et informelles de l'État est au cœur du modèle de vitalité (Figure.1). En conséquence, la position acquise dans la hiérarchie sociale par les membres d'une communauté linguistique influence le rôle attribué à la langue de ce groupe pour les fonctions sociales hautes et basses, ce qui correspond à la variable "statut linguistique» du modèle de la vitalité linguistique (Figure.1).

Dans le cadre de la vitalité, la transmission intergénérationnelle faisait partie des facteurs démographiques ou plus exactement du nombre absolu de locuteurs utilisant encore une certaine langue comme langue maternelle ou langue seconde. Parmi les variables influençant l'augmentation ou la diminution du nombre des locuteurs de cette langue, le modèle de la vitalité incluait d'autres facteurs importants à savoir le taux de natalité, les mariages mixtes, l'immigration et l'émigration. Or, il est évident que la transmission intergénérationnelle de la langue maternelle (L1) devrait constituer un facteur distinct de la vitalité linguistique, contribuant au poids démographique de telle ou telle communauté linguistique.

Tout comme on peut améliorer le modèle de vitalité grâce au modèle RLS (Reversing language shift : Inversion du glissement linguistique), l'inverse est tout aussi vrai. Le modèle RLS peut être enrichi en tenant compte de la gamme complète des variables utilisées pour évaluer la vitalité relative des communautés linguistiques en contact. Il est clair d'après l'exemple québécois que le modèle de vitalité peut servir d'instrument d'analyse plus systématique pour mieux cibler les efforts de RLS et peut préciser ce qui reste implicite dans le modèle de RLS de Fishman (1991). Par exemple, il n'est pas certain qu'une analyse limitée au modèle RLS pour évaluer la situation du français au Québec dans les années 1970 aurait conclu que l'aménagement linguistique en faveur du français était vraiment nécessaire. Comme nous l'avons vu, la transmission intergénérationnelle du français au sein de la majorité francophone était pratiquement intacte à cette époque. Cependant, c'est le constat de la faiblesse du statut du français sur d'autres variables de vitalité telles que le contrôle institutionnel (langue de travail) et la démographie (déclin de la fécondité, anglicisation des immigrants) qui a servi de catalyseur pour convaincre la majorité francophone qu'il était temps d'élaborer des politiques linguistiques afin d'(inverser le glissement linguistique») et assurer la pérennité du fait français au Québec. Il paraît donc judicieux de considérer le modèle de vitalité comme un complément nécessaire à l'échelle de discontinuité intergénérationnelle de Fishman (1991) plutôt que d'y voir simplement l'inverse de l'échelle GIDS. En fait, le modèle de vitalité peut s'avérer fort utile comme outil d'analyse sociolinguistique de plusieurs types de modèles d'aménagement linguistique autres que celui de Fishman (1991, 1999).

La façon de conceptualiser la vitalité linguistique débouche sur une autre dimension susceptible de contribuer au modèle RLS. Bourhis, Giles et Rosenthal (1981) ont proposé que les membres d'un groupe linguistique se formaient une impression subjective de la force de leur communauté linguistique dans la structure sociale. Cette perception subjective de la vitalité endogroupe est plus ou moins analogue aux analyses de vitalité proposées par les experts en psychologie sociale et en aménagement linguistique. Le questionnaire de vitalité subjective (SVQ) fut donc mis au point afin de jauger les évaluations des membres d'un groupe pour chacune des composantes des variables du modèle de vitalité objective : contrôle 
institutionnel, poids démographique et statut (Bourhis et al., 1981). Un certain nombre d'études utilisant ce questionnaire ont montré que dans beaucoup de contextes, les membres d'une communauté linguistique avaient une perception subjective de leur vitalité endogroupe plus ou moins équivalente à celle établie d'après des mesures objectives (Bourhis et Sachdev, 1984 ; Allard et Landry, 1994). Dans d'autres cas, les études ont montré que les évaluations subjectives de vitalité étaient systématiquement biaisées. Harwood, Giles et Bourhis (1994) ont montré que ces perceptions erronées étaient souvent à l'avantage de la vitalité de l'endogroupe mais, dans certains cas aussi, à l'avantage de la vitalité de l'exogroupe. Néanmoins, de telles distorsions ne portent pas sur les écarts les plus évidents de la vitalité des communautés linguistiques comparées. Elles ne concernent que des éléments pour lesquels l'écart objectif entre l'endogroupe et l'exogroupe est marginal. Sachdev et Bourhis (1993) ont montré que des variables d'ordre cognitif et motivationnel peuvent servir à expliquer les écarts dans les évaluations de vitalité subjectives concernant l'endogroupe et l'exogroupe.

En outre, Allard et Landry (1986) ont suggéré que les perceptions de vitalité subjective peuvent servir à prédire les comportements langagiers si l'on tient compte non seulement des croyances générales sur la vitalité de groupe (SVQ, Bourhis et al., 1981) mais aussi des croyances influencées par les aspirations des répondants et de leur volonté de se mobiliser afin d'améliorer la vitalité de leur endogroupe sur le plan démographique ainsi que sur le plan du contrôle institutionnel et du statut (croyances personnelles, BEVQ). Constatant la faible vitalité de leur endogroupe sur le plan du soutien institutionnel, certains expriment le désir (BEVQ) d'agir collectivement afin de créer les écoles nécessaires pour assurer l'enseignement de la langue minoritaire. En revanche, d'autres admettent que la vitalité de leur endogroupe est faible, voire en déclin (SVQ) et expriment des croyances et des aspirations (BEVQ) qui trahissent une absence de motivation pour redresser la situation par une action individuelle ou collective; parfois, le déclin de la vitalité de leur propre groupe linguistique leur est totalement indifférent. Au Canada, Allard et Landry (1994) ont montré qu'une combinaison des deux types de croyances : générales (SVQ) et liées aux aspirations personnelles (BEVQ) était un meilleur prédicteur des comportements langagiers tels que la transmission intergénérationnelle ou la volonté de scolariser son enfant dans sa langue minoritaire plutôt que dans la langue de la majorité dominante.

Les membres d'un groupe perçoivent-ils l'échelle de discontinuité intergénérationnelle (GIDS) de la même façon que les experts en aménagement linguistique? Il est possible de mettre au point une échelle GIDS «subjective» pour évaluer dans quelle mesure les militants et les simples membres d'une minorité linguistique sont conscients de la gravité de la non transmission intergénérationnelle de leur langue endogroupe. Les évaluations subjectives pourraient être recueillies sur des échantillons composés d'experts et de membres de la population concernée avant de formuler les plans d'intervention pour «inverser le glissement linguistique». En tenant compte des données démolinguistiques des recensements et des enquêtes sociolinguistiques, les perceptions subjectives de vitalité (SVQ ; BEVQ) et de GIDS pourraient aider à peaufiner les étapes ultérieures du plan d'aménagement linguistique dont le but est d'inverser le glissement linguistique. Une combinaison de données objectives et subjectives concernant la vitalité et le GIDS d'une minorité linguistique en difficulté constitue une méthode plus précise 
pour planifier et mettre sur pied une politique linguistique visant à inverser le glissement linguistique (RLS) dans le cadre du modèle GIDS de Fishman (1991 ; 2001).

\section{3) Bilan de 20 années d'aménagement linguistique au Québec}

Les changements sociaux découlant de la "Révolution tranquille» des années 1960 ont exacerbé la conscience des francophones sur la menace diglossique qui planait sur le français en raison de sa co-existence avec l'anglais, langue de prestige, des affaires et de la mobilité sociale (Rocher, $1992 ; 2000$ ). Comme l'illustre le livre Nègres blancs d'Amérique (Vallière, 1969), il devenait urgent pour la majorité francophone issue de la classe ouvrière de résister à la main mise des anglophones sur l'économie du Québec et de réagir contre «l'apathie de la petite bourgoisie» franco-québécoise. Étant donné le déclin de son passé rural et religieux, la modernisation de la société québécoise signifiait aussi que le français représentait le dernier symbole de l'identité québécoise dans une société de plus en plus orientée vers le matérialisme et la consommation (Sachdev et Bourhis, 1990; Thériault, 2000). Puisque le français constituait le dernier bastion de la spécificité québécoise face à la force d'attraction de la langue et de la culture anglo-américaine, il était sans doute inévitable que les gouvernements successifs au Québec estiment nécessaire de légiférer dans le domaine de la langue (Bourhis et Lepicq, 1993; Woehrling, 2000).

Comme nous l'avons déjà mentionné auparavant, dans les années 1970, le français se trouvait généralement en bonne position au Québec pour ce qui est de la démographie et du contrôle institutionnel ; en outre, sa transmission intergénérationnelle était relativement bien assurée (Fishman, 1991). Toutefois, les activistes et les intellectuels québécois réussirent à canaliser l'attention des francophones sur des aspects plus problématiques de leur destin en tant que minorité francophone en Amérique du Nord. Parmi les facteurs susceptibles de compromettre l'avenir du français au Québec, on invoquait : 1) le déclin des francophones dans les provinces anglophones du Canada, 2) la baisse du taux de fécondité de la population francophone, 3) le choix des immigrants de scolariser leurs enfants dans le système anglophone plutôt que francophone, 4) La domination par les anglophones de l'économie québécoise (d'Anglejan 1984 ; Laporte, 1984). Entre 1969 et 1996, les gouvernements successifs au Québec ont promulgué une série de lois linguistiques portant sur chacun des points risquant de compromettre l'avenir du français dans la province (loi 63,1969 ; loi 22,1974 ; loi 101, 1977 ; loi 57,1983 ; loi 142,1986 ; loi 178,1988 ; loi 86,1993 ; loi 40,1997 ; loi 170,171 en 2000 ; Woehrling, 2000). Jusqu'à maintenant, la Charte de la langue française (loi 101) demeure la plus importante de ces lois (Bourhis, 1984a). Selon le concepteur de la loi 101, le regretté Camille Laurin, l'objectif de la loi n'était pas seulement de neutraliser les menaces risquant de compromettre la pérennité du français mais aussi de «remédier aux injustices et humiliations passées subies par mes concitoyens dans mon village et partout au Québec» (dernier discours de Camille Laurin, 11 décembre 1998).

La loi 101 garantissait à chaque Québécois le droit de pouvoir communiquer en français dans ses rapports avec l'administration civile, les organismes semi- 
publics et les entreprises et assurait à tous les clients le droit de recevoir des renseignements et des services en français. La loi garantissait aussi le droit à tous les employés de travailler en français et de ne pas être licenciés ou déclassés sous prétexte qu'ils étaient unilingues et francophones. En ce qui concerne la langue de travail, la loi 101 appliquait pour l'essentiel des mesures déjà en vigueur depuis la loi 22. Les entreprises de plus de 50 employés étaient obligées de se soumettre à l'obtention d'un certificat de francisation attestant qu'elles possédaient l'infrastructure nécessaire pour utiliser le français comme langue de travail dans leur fonctionnement (Daoust, 1984 ; Bouchard, 1991). A partir de 1996, le certificat de francisation fut obligatoire pour les entreprises désireuses de traiter avec le gouvernement provincial.

La loi 101 garantissait aussi le droit d'être scolarisé en anglais à tout élève anglo-Québécois. Tous les enfants d'immigrants déjà inscrits dans des écoles anglaises lors de la promulgation de la loi ainsi que leur fratrie étaient aussi assurés de l'accès au système scolaire anglophone. Néanmoins, la loi spécifiait que tous les immigrants subséquents au Québec étaient obligés d'inscrire leurs enfants dans le système scolaire francophone élémentaire et secondaire. Il faut cependant préciser que la loi n'affectait pas la liberté du choix de la langue au niveau primaire et secondaire pour les parents qui désiraient envoyer leurs enfants dans les écoles privées payantes. En outre, la liberté du choix de la langue s'appliquait à tout étudiant de niveau post secondaire qui avait le choix entre CEGEP et universités francophones ou anglophones au Québec. La loi 101 incluait enfin une clause controversée qui bannissait les langues autres que le français du «paysage linguistique», à savoir la signalisation routière, l'affichage officiel émanant du gouvernement et les enseignes commerciales (Bourhis et Landry, 2002).

Tout en incluant des dispositions relatives à l'aménagement du corpus, la loi avait pour objectif primordial d'améliorer le statut du français par rapport à l'anglais dans la société québécoise (Bourhis et Lepicq, 1993). Les relations de cause à effet sont difficiles à établir quand on évalue l'impact des politiques linguistiques sur les comportements langagiers et l'inversion du glissement linguistique. Le cas du Québec n'échappe pas à la règle et il faut donc tenir compte de cette limitation dans la présentation des faits et des données qui suivent.

\section{A) La loi 101 et l'évolution démolinguistique}

Bien des francophones réagirent de façon positive à la promulgation de la loi 101 dont on percevait l'efficacité pour protéger l'avenir linguistique de la majorité francophone de la province (Bourhis, 1984b ; Levine, 1990 ; Maurais, 1987). Sur le plan démographique, la proportion de locuteurs du français langue maternelle par rapport à la population totale du Québec passa de $80,7 \%$ en 1971 (4 866 $410)$ à $81,5 \%(5741438)$ en $1996^{3}$. L'usage du français au foyer augmenta, passant de $80,8 \%(4870100)$ en 1971 à $81,8 \%(5830082)$ en 1996 . Considẻrés dans leur ensemble, ces chiffres suggèrent une amélioration de la transmission intergénérationnelle du français entre 1971 et 1996. Ainsi, les résultats du

\footnotetext{
Dans cette section et les suivantes, les données statistiques se réfèrent aux résultats du recensement de 1996 pour lequel on dispose d'analyses détaillées. Te recensement le plus récent date de 2001 mais Statistique Canada n'a pas encore produit les données pertinentes à notre analyse.
} 
recensement de 1996 laissent présager un changement de langue en faveur du français comme le suggèrent les scores plus élevés de l'usage du français au foyer par rapport au nombre de locuteurs du français langue maternelle du recensement. Bien que cette tendance puisse s'expliquer en partie par des innovations récentes dans la méthodologie du recensement, le changement est imputable aux allophones plus qu'aux anglophones, les allophones ayant opté pour le français comme langue du foyer (Castonguay, 1998). Cette tendance favorable au français risque d'être neutralisée par le faible taux de fécondité de la population francophone du Québec qui, de 4.2 enfants par femme en 1961, était seulement de 1,6 en 1996. Castonguay (1998) conclut à ce propos : «une fécondité insuffisante pendant plus de vingt ans préfigure un déclin en chiffres absolus de la population francophone du Québec - a fortiori du Canada dans son ensemble - à compter du prochain millénaire» (p. 44).

L'augmentation du bilinguisme français-anglais chez les francophones reflète l'intégration croissante des francophones du Québec au cadre économique et culturel nord-américain. Tandis que seulement $26 \%$ des locuteurs de langue maternelle française se déclaraient bilingues en 1971, cette proportion était de 34 $\%$ en 1996. Cette augmentation du nombre des bilingues d'origine francophone est surtout évidente à Montréal où il demeure avantageux de parler le français et l'anglais pour effectuer des échanges économiques et culturels avec des partenaires locaux, canadiens et internationaux.

Les anglophones, en général, se montrèrent hostiles à la loi 101 dans la mesure où elle constituait à leurs yeux une atteinte au statut traditionnel d'élite de la minorité anglophone de la province (Legault, 1992 ; Scowen, 1991 ; Stevenson, 1999). La loi 101 obligea beaucoup d'anglophones à se considérer comme faisant partie d'une communauté minoritaire plutôt que comme membres d'une élite dominante (Caldwell 1984, 1994, 1998). Suite à l'élection du Parti Québécois en 1976, beaucoup d'anglophones mécontents des politiques linguistiques et fiscales du Québec émigrèrent en Ontario et dans d'autres provinces canadiennes (Caldwell, 1984). En fait, l'émigration hors de la province et le déclin du taux de fécondité furent les principaux facteurs de l'érosion de la vitalité démographique des anglophones du Québec (Castonguay, 1998, 1999). D'après les données du recensement, on enregistre un déclin de $12 \%$ de la population de langue maternelle anglaise au Québec entre1971 et 1981 (Caldwell, 1984). Cette perte sèche de 158 000 locuteurs de l'anglais langue maternelle concerne les couches économiques les plus mobiles de la population anglophone, pour la plupart des unilingues d'ascendance britannique âgés d'une vingtaine d'années et diplômés d'université. Dans les années 1990, ces tendances migratoires eurent un effet dévastateur sur la proportion d'anglophones au Québec : de $13 \%$ en 1971 (789 000), la proportion des locuteurs de langue maternelle anglaise était tombée à 8,8\% en 1996 (622 000), c'est-à-dire une perte de 167000 anglophones pour la province. L'usage de l'anglais au foyer passa de 14,7\% en 1971 (887 875) à 10,8\% en 1996 (762 457). Pourtant, en 1996, la différence entre l'usage de l'anglais au foyer $(10,8 \%)$ et la proportion de locuteurs de l'anglais langue maternelle $(8,8 \%)$ témoigne du remarquable pouvoir d'attraction de l'anglais au Québec même vingt ans après la mise en vigueur de la loi 101 (Castonguay, 1997, 1999). Les analyses des résultats des recensements de 1971 à 1996 ont montré que les allophones sont responsables de ce changement de langue en faveur de l'anglais au foyer, non seulement au Québec mais aussi dans tout le Canada (Castonguay, 1999). Précisons finalement 
que l'adoption de la loi 101 et l'exode d'un nombre substantiel d'anglophones unilingues ont eu un effet sur la proportion de Québécois anglophones déclarant connaître le français. Ainsi, chez les anglophones du Québec, la connaissance de la langue française a progressé de $37 \%$ en 1971 à $63 \%$ en 1996.

Malgré un taux optimal de transmission intergénérationnelle, il est indéniable que la minorité anglophone dù Québec connaît un fort déclin d'après des indicateurs plus fondamentaux de vitalité démographique tels que les chiffres absolus et relatifs, la migration hors de la province et les taux de fécondité. Étant donné que ce taux passa de 3,3 enfants par femme en 1961 à seulement 1,6 en 1996 et que les chances d'une immigration substantielle en provenance des provinces anglo-canadiennes étaient minces, les anglophones du Québec durent compter de plus en plus sur les stratégies d'intégration linguistique des allophones et des immigrants internationaux qui venaient s'établir dans la province.

Au Canada, le terme langues patrimoniales ou langues d'origine sert à désigner les langues autres que les deux langues officielles : l'anglais et le français. Au Québec, le nivellement graduel de la force d'attraction du français et de l'anglais grâce à la loi 101 a fait du maintien de la langue et de la culture d'origine une option plus attirante pour les allophones et les communautés immigrantes de la Province (Bourhis, 1994b). Les résultats du recensement de 1996 ont montré que les allophones constituaient 9,3\% de la population totale du Québec. Sur ces 681 780 allophones, les groupes linguistiques les plus importants sont : les Italiens (20 $\%$ ), les Espagnols (10\%), les Arabes $(8,8 \%$ ), les Grecs $(6,5 \%)$, les Chinois $(6,2$ $\%$ ) et les Portugais ( $5 \%$ ). Les analyses des données du recensement de 1986 avaient indiqué que le maintien de la langue d'origine était plus fort chez les allophones du Québec que chez les allophones résidant dans les provinces anglophones du Canada (Pendakur, 1990). Ainsi, en 1986, $68 \%$ des immigrants de première génération établis au Québec déclaraient qu'ils utilisaient encore leur langue d'origine comme seule langue de communication à la maison (63\% en 1991) par rapport à $48 \%$ de ceux établis hors Québec. Cette différence du maintien des langues d'origine était encore plus marquée dans le cas des allophones de deuxième génération au Québec (63\%) comparés à leurs congénères ailleurs au Canada (34\%). Les données du recensement de 1996 indiquent que les allophones du Québec demeurent plus enclins à maintenir l'usage de leur langue d'origine à la maison que les allophones établis dans le reste du Canada. Ce maintien de la langue patrimoniale constitue un capital linguistique pour le Québec synonyme d'enrichissement culturel ; en outre, il ouvre des perspectives sur le plan des échanges commerciaux avec les pays d'origine de ces immigrants multilingues.

Suite à des accords conclus avec le ministère de l'Immigration canadien entre 1978 et 1991, le gouvernement du Québec a acquis un certain niveau de contrôle sur la sélection des immigrants d'après les besoins linguistiques et de main d'œuvre spécifiques à la Province. Dans les deux dernières décennies, le ministère québécois des Affaires civiques et de l'Immigration est parvenu à attirer des immigrants ayant une connaissance du français, dont certains originaires des ex-colonies françaises ainsi que des immigrants dont la connaissance d'une langue romane (par exemple l'espagnol) pouvait faciliter l'apprentissage du français. Des études ont montré qu'une certaine connaissance du français encourage les immigrants à s'intégrer à la communauté d'accueil francophone alors qu'une connaissance de l'anglais prédispose les immigrants à s'intégrer à la minorité d'accueil anglophone (Veltman, 
1998). De toute évidence, le gouvernement du Québec s'efforce de recourir au contrôle institutionnel dont il dispose pour sélectionner les allophones et les immigrants et encourager leur intégration à la majorité d'accueil francophone plutôt qu'à la minorité anglophone.

Des tensions linguistiques croissantes entre les communautés d'accueil francophone et anglophone ont exercé des pressions sur les minorités allophones pour qu'elles prennent ouvertement parti dans le débat linguistique et politique au Québec (Bourhis, 1994b). Une façon pour les allophones de faire face à la situation fut d'apprendre à la fois le français et l'anglais, ce qui a fait passer le bilinguisme officiel de $33 \%$ en 1971 à $48 \%$ en 1996. Vu qu'une forte proportion d'allophones au Québec conservent une certaine connaissance de leur langue d'origine même à la deuxième génération, il s'ensuit que beaucoup d'entre eux sont trilingues, ce qui contribue à la richesse linguistique et culturelle de la province. En comptabilisant les allophones qui connaissent seulement le français ou les deux langues officielles, les résultats du recensement montrent que la proportion d'allophones qui déclaraient connaître le français est passée de $47 \%$ en 1971 à $71 \%$ en 1996. En contrepartie, la proportion d'allophones déclarant connaître l'anglais a baissé entre 1971 et 1996 de $70 \%$ à $66 \%$. En fin de compte, la loi 101 conjuguée à une immigration en provenance de pays de langues romanes a eu un retentissement sur le transfert linguistique des immigrants récents. Parmi les allophones qui s'établirent dans la province avant 1976, seulement $34 \%$ avaient adopté le français comme langue du foyer alors que parmi ceux arrivés après la loi 101, (entre 1976 et 1991) $67 \%$ utilisaient le français comme une des langues de la maison (Québec, 1996a). Bien que la «langue du foyen» ait servi d'indice pour mesurer indirectement l'impact de la loi 101 sur les usages linguistiques, précisons que cette loi ne concernait que l'usage de la langue dans la sphère publique (relations professionnelles, commerciales et administratives). Il n'a jamais été question de légiférer sur l'usage des langues dans la sphère privée, par exemple au foyer ou dans les conversations entre amis.

\section{3b) Montréal : enjeu ultime de la francophonie en Amérique du Nord}

Au milieu du $19^{\mathrm{imc}}$ siècle, Montréal était une ville majoritairement anglophone. Montréal devint progressivement francophone surtout à partir du début du $20^{\text {ème }}$ siècle, période à partir de laquelle la communauté anglophone amorça un déclin inexorable. L'urbanisation et la prolétarisation des francophones du Québec changèrent le profil démographique de la ville et, dans les années 1950, Montréal était devenue une ville dont la population était majoritairement francophone. Aujourd'hui, la région métropolitaine de Montréal est la mégapole du Québec avec 3,4 millions d'habitants sur une population totale de 7.2 millions au Québec. La proportion des locuteurs de langue maternelle française dans la grande région de Montréal incluant les couronnes nord et sud de l'île a augmenté : de $65 \%$ en 1951 à $70 \%$ en 1986 avec un léger recul en $1996(67 \%)$. En contrepartie, la proportion des locuteurs de langue maternelle anglaise dans cette grande région de Montréal a baissé : de $26 \%$ en 1951 à $17 \%$ en 1986 pour arriver à $13 \%$ en 1996. Depuis les années 1950, l'arrivée des immigrants allophones a été décisive pour transformer le 
profil démolinguistique de la grande région de Montréal. La proportion des allophones a augmenté : de $8,6 \%$ en 1951 à $13 \%$ en 1986 et à $20 \%$ en 1996 .

Tout en étant multiculturelle et multilingue à l'échelle de sa grande région, il n'en reste pas moins que la partie ouest de l'île de Montréal est majoritairement anglophone alors que les francophones forment la majorité dans les régions à l'est de l'île avec une diversité considérable de communautés culturelles dans les régions au centre de l'île de Montréal. Comptant presque la moitié de la population du Québec, la grande région de Montréal, ville multilingue, reste tout à fait différente des autres régions unilingues francophones de la province. Les autres centres urbains sont homogènes sur le plan linguistique avec une présence de francophones natifs comptant pour plus de $90 \%$ (ville de Québec, Trois-Rivières, Sherbrooke) à $80 \%$ de la population (région de Hull). Les régions rurales de la province sont aussi majoritairement francophones avec $92 \%$ de la population déclarant le français comme langue maternelle.

Quand on analyse la répartition des immigrants et des allophones à travers le Québec, on remarque que la grande majorité ( $88 \%$ ) habitent la grande région de Montréal, constituant ainsi $16 \%$ du total de la population métropolitaine. Les communautés allophones et immigrantes se concentrent sur l'île de Montréal parce que la ville offre de meilleures perspectives économiques et leur permet d'entretenir des réseaux endogroupe qui foumissent le soutien culturel, linguistique et économique nécessaire pour s'adapter plus facilement à la société d'accueil. La concentration des communautés issues de l'immigration dans la métropole signifie que ce sont les populations d'accueil de Montréal plutôt que celles des autres régions du Québec qui ont dû s'adapter aux réalités du multilinguisme et du pluralisme culturel dans la province.

Étant donné le rôle crucial de Montréal dans l'économie du Québec, certains francophones estiment que c'est dans cette ville que le sort du français se jouera en Amérique du Nord. C'est la nécessité d'utiliser le français pour communiquer avec la majorité francophone unilingue qui a justifié l'aménagement linguistique en faveur du français par rapport à l'anglais au Québec. En revanche, si la majorité des francophones et des allophones de Montréal deviennent bilingues, alors la nécessité d'utiliser le français ne constituera plus un argument valable face aux avantages d'utiliser l'anglais comme seule langue des affaires et de la mobilité sociale de la ville dans un contexte nord-américain. Chez les locuteurs du français langue maternelle dans la grande région de Montréal, la proportion de bilingues (français/anglais) a augmenté, passant de $38 \%$ en 1971 à $44 \%$ en 1986 et à $47 \%$ en 1996. De même, le nombre des locuteurs anglophones de naissance qui se sont déclarés bilingues a augmenté, passant de $35 \%$ en 1971 à $54 \%$ en 1986 et à $62 \%$ en 1996. La proportion des allophones de Montréal qui déclarent connaître à la fois le français et l'anglais a augmenté, de $35 \%$ en 1971 à $50 \%$ en 1986 avec un léger recul en $1996(49 \%)$.

Certains francophones qui militent pour la cause du français considèrent que le bilinguisme individuel représente une menace potentielle au statut du français, langue de la majorité à Montréal. En revanche, pour d'autres, le bilinguisme constitue un atout qui peut favoriser le dynamisme culturel et économique de la ville. Les bilingues et les trilingues de la catégorie des jeunes adultes, qu'ils soient d'origine francophone, anglophone et allophone ont tous été scolarisés au moment où la loi 101 était plus ou moins acceptée comme un fait accompli. Ayant fréquenté 
le CEGEP ou l'université, ces jeunes bilingues se côtoient dans le milieu du travail et des loisirs et ont recours à des stratégies de communication interculturelle y compris des permutations de code entre le français et l'anglais qui se stabilisent pour former un répertoire communicatif bilingue (convergence linguistique réciproque, Amiot et Bourhis, 1999 ; Moise et Bourhis, 1994). Tout en restant fidèles à leur communauté linguistique d'origine, ces «caméléons linguistiques» se forgent des identités bi ou multiculturelles auxquelles ils ont recours selon les circonstances, les interlocuteurs et les besoins des échanges transculturels. Bien que ces observations ne soient pas applicables à l'ensemble de la population bilingue de Montréal, il est vrai que des sous groupes de jeunes bilingues et trilingues d'origine allophone et anglophone redéfinissent les relations entre le français et l'anglais dans un contexte post-moderme et d'une façon moins conflictuelle que les Montréalais des générations précédentes qui se sont investis dans les luttes linguistiques qui ont abouti à l'adoption des lois linguistiques à l'assemblée nationale du Québec.

Quel a été l'impact des lois linguistiques favorisant le français par rapport à l'anglais au Québec? Dans une enquête téléphonique effectuée en 1997, le Conseil de la Langue française (CLF) a cherché à évaluer les effets de la loi 101 sur l'utilisation du français et de l'anglais en tant que langues de la vie publique dans des contextes tels que les centres commerciaux, les magasins, les banques, les hôpitaux et avec les fonctionnaires au niveau local et provincial (Béland, 1999). Un vaste échantillon représentatif de francophones, d'anglophones et d'allophones fut interviewé à travers la province y compris dans la région du grand Montréal. En combinant les situations d'utilisation de la langue en une seule variable portant l'étiquette «langue publique», les résultats ont montré un usage soutenu du français à la fois chez les francophones et les allophones mais moins fréquent chez les anglophones (cf. Moise et Bourhis, 1994). Dans le grand Montréal, les résultats ont montré que $97 \%$ des francophones déclaraient l'utilisation exclusive ou presque du français dans ces contextes publics tandis que $3 \%$ seulement déclaraient utiliser principalement l'anglais. La majorité des allophones (54\%) déclaraient utiliser exclusivement ou presque toujours le français dans les endroits publics tandis que $39 \%$ déclaraient utiliser principalement l'anglais et seulement $8 \%$ principalement leur langue d'origine. Considérés dans leur ensemble, ces résultats suggèrent que le français est devenu la langue habituelle de communication publique pour les francophones de Montréal alors que pour les allophones, le français gagne du terrain par rapport à l'anglais. En revanche, seulement $23 \%$ des anglophones rapportaient utiliser principalement le français dans ces endroits publics tandis que $77 \%$ déclaraient l'usage exclusif ou presque de l'anglais. De toute évidence, les anglophones du Québec, quelle que soit leur compétence dans les deux langues, peuvent encore se permettre d'utiliser l'anglais comme langue publique surtout dans l'ouest de Montréal, tendance que l'on retrouve chez les anglophones dans des régions de la province autres que celle de Montréal (Landry, Allard et Bourhis, 1987).

Les anglophones de l'ouest de l'île de Montréal se distinguent des francophones non seulement sur le plan linguistique mais aussi politique, notamment pour ce qui est de la «question nationale». Ainsi, lors du référendum sur l'indépendance du Québec le 30 octobre 1995, plus de $90 \%$ des anglophones et des allophones ont voté contre la souveraineté du Québec, favorisant ainsi le maintien du Québec au sein de la fédération canadienne. Les autochtones du Québec 
(Amérindiens et Inuits) refusèrent quant à eux de participer au vote référendaire du gouvernement québécois. En revanche, dans le cadre de leur propre référendum, plus de $95 \%$ d'entre eux votèrent contre le projet souverainiste, privilégiant ainsi l'option canadienne (Grand Council of the Crees, 1998). Le soit de la défaite du vote souverainiste qui ne tenait qu'à un fil $(49,4 \%$ de oui contre $50,6 \%$ de non), le Premier ministre du Québec, Jacques Parizeau, s'adressa aux adeptes du Parti québécois partisans de la souveraineté, en ces termes:

"Aye, si vous voulez là, on va arrêter de parler des francophones du Québec, voulez-vous. On va parler de nous. A 60 pour cent, on a voté pour... C'est vrai qu'on a été battus au fond par quoi? par l'argent, pis des votes ethniques, essentiellement. Alors, ça veut dire que la prochaine fois, au lieu d'être 60 ou 61 pour cent à voter oui, on sera 63 ou 64 et puis ça suffira» (La Presse, 2 novembre 1995, B3).

En novembre 1997, le même Jacques Parizeau qui n'était plus Premier ministre depuis sa résignation au lendemain du vote référendaire, précisa l'origine du «vote ethnique» de sa déclaration précédente : «J'ai dit à plusieurs reprises que le Congrès juif du Canada, le Congrès grec du Canada et le Congrès italien mènent une très bonne bataille contre la souveraineté (La Presse, 26 novembre 1997, B8).

Ces déclarations contribuèrent à la polarisation ethnique de la société québécoise tout en aggravant le sentiment d'exclusion des allophones et des anglophones de la province. Lors d'un sondage effectué en 1996, plus de $80 \%$ des non-francophones du Québec (anglophones et allophones) se montrèrent favorables à la "partition» qui permettrait aux régions majoritairement anglophones de la province de rester au sein de la fédération canadienne au cas où le vote majoritaire en faveur de la séparation l'emporterait à l'avenir (l'Actualité, 1996). En revanche, en pareil cas, les trois quarts des francophones étaient hostiles à la partition du Québec. La plupart des anglophones et des allophones favorables à la partition résidaient dans les municipalités à statut bilingue de l'ouest de l'île de Montréal. Durant les années 1996-1997, les assemblées de ces municipalités majoritairement anglophones votèrent pour la partition de leur municipalité dans l'éventualité d'un scrutin favorable à la séparation du Québec. Précisons que ces votes municipaux n'ont pas de statut légal ou juridique puisque la Constitution canadienne et l'Acte de l'Amérique du Nord donnent à chacune des provinces le droit de créer, d'amalgamer ou d'éliminer les municipalités situées sur leur territoire. Néanmoins, la visibilité nord-américaine de ces municipalités anglophones favorables à la partition risquait de jeter le discrédit sur le gouvernement du Parti québécois en cas de référendum favorable à l'indépendance.

En décembre 2000, sous prétexte de réduire les dépenses gouvernementales, le Gouvernement du Parti québécois adopta la loi 170 sur les fusions municipales. Cette loi visait à amalgamer plusieurs municipalités du Québec, notamment celles situées sur l'île de Montréal (Schnobb, 2001). Or, plusieurs recherches en urbanisme tant en Amérique du Nord qu'en Europe montrent que les fusions municipales ont rarement pour effet de réduire les dépenses de l'Etat ou de répondre plus efficacement aux besoins et aux aspirations des citoyens (Sancton, 2000). La loi 170 prévoyait la fusion de 28 municipalités de l'île de Montréal, dont les quinze municipalités majoritairement anglophones situées à l'ouest de l'île de Montréal. Cette fusion de municipalités anglophones ayant un statut bilingue conformément à la loi 101 signifiait la mise en minorité des anglophones au sein de 
la nouvelle Ville de Montréal «Une île, une ville». A souligner: seule la ville de Montréal fut gratifiée d'un statut linguistique précisé dans le texte de loi : «Montréal est une ville de langue française» (loi 170, article 1, Québec 2000). Ainsi, le Gouvernement du Québec, occultant la réalité sociolinguistique de l'île de Montréal, la consacrait officiellement et uniquement de langue française. En conséquence, les quinze villes à l'ouest de Montréal perdirent leur «statut bilingue» qui, avant la loi 170, garantissait des services municipaux en anglais et en français à leurs résidents. Après l'adoption de la loi 170, les services bilingues des anciennes villes transformées en «arrondissements de quartiers» devinrent facultatifs. La loi 170 fut adoptée en dépit de la résistance et des manifestations de nombreux citoyens anglophones, allophones et francophones inquiets du déficit démocratique et identitaire causé par la disparition de leur ville. Ainsi, un sondage à l'échelle de Montréal effectué en décembre 2000 montrait que $80 \%$ de la population anglophone et $61 \%$ de la population allophone étaient opposés à la fusion (étaient en faveur : $9 \%$ d'anglophones et $23 \%$ d'allophones).En revanche, la majorité francophone de Montréal était divisée sur la question : $40 \%$ étaient en faveur de la fusion et $39 \%$ y étaient hostiles.

Pour éviter d'avoir à octroyer le statut bilingue à de nouvelles municipalités, le Gouvernement du Parti québécois adopta la loi 171 en décembre 2000. Le «statut bilingue», conformément à la loi 101 , pouvait être attribué à une ville à condition que plus de la moitié des citoyens résidents $(50 \%+1)$ aient une langue maternelle autre que le français selon les données du recensement canadien (anglophones + allophones). Ce statut octroyait à une ville le droit de garantir des services municipaux en français et en anglais afin de servir les citoyens dans la langue de leur choix. Or la loi 171 stipule que le statut bilingue ne peut être accordé que si une majorité des citoyens $(50 \%+1)$ ont l'anglais comme langue maternelle. Étant donné le déclin de la minorité anglophone du Québec et la faible proportion d'immigrants de langue maternelle anglaise, il était évident qu'aucune ville ou arrondissement du Québec ne pourrait accéder au statut bilingue après l'adoption de la loi 171.

La loi 170 fut contestée juridiquement par deux groupes distincts durant l'année 2001 : celui des villes à statut bilingue et celui des villes à statut francophone (voir Tableau 1). L'argumentaire de la contestation juridique des villes à statut francophone est que les fusions forcées modifient de manière irréversible la liberté de choisir une ville en fonction du cadre de vie qu'elle offre (Schnobb, 2001). Les citoyens ayant choisi d'habiter les villes distinctes à démocratie de proximité voient leur liberté de choix annulée par la fusion forcée de leur ville au sein de la mégapole du grand Montréal post-fusion. L'argumentaire de la contestation juridique des villes anglophones à statut bilingue se résume ainsi : les fusions forcées portent atteinte aux droits linguistiques de la minorité anglophone qui perd le seul niveau de gouvernement qu'elle contrôle au Québec. Les arrondissements à statut bilingue qui ont le droit d'offrir des services en anglais sont des coquilles vides ayant aucun statut juridique et sont contrôlés par le Conseil municipal du Grand Montréal majoritairement francophone, ville dont le seul statut linguistique officiel est "Montréal est une ville de langue française" (article 1, Loi 170). Suite à un méga procès hautement médiatisé, le juge de première instance du Québec ainsi que la cour d'appel supérieure de la province ont rejeté ces deux argumentaires en invoquant la Constitution canadienne qui donne aux 
gouvernements provinciaux l'entière juridiction sur la création, la fusion et l'abolition de toutes villes sur leur territoires. Tout en reconnaissant que la communauté anglophone du Québec perdait une part importante de leur support institutionnel en tant que minorité linguistique au Québec, les jugements reconnurent la constitutionalité de la loi 170 fusionnant les villes à statut bilingue de Montréal.

Tableau 1 : Contestation juridique de la loi 170 s'appliquant à la Ville de Montréal selon le statut linguistique des villes et la langue maternelle des citoyens.

\begin{tabular}{|l|c|c|c|c|}
\hline \multirow{2}{*}{$\begin{array}{c}\text { Loi 170 sur les fusions } \\
\text { municipales adoptée en } \\
\text { décembre 2000 et contestée à } \\
\text { Montréal en 2001 }\end{array}$} & $\begin{array}{c}\text { Population } \\
\text { totale de ces } \\
\text { villes }\end{array}$ & \multicolumn{3}{|c|}{$\%$ langue matemelle } \\
\cline { 3 - 5 } $\begin{array}{l}\text { Villes à statut bilingue qui ont } \\
\text { contesté juridiquement la loi } \\
170 \text { (12/14) }\end{array}$ & 218,127 & $22 \%$ & $54 \%$ & $22 \%$ \\
\hline $\begin{array}{l}\text { Villes à statut bilingue qui } \\
\text { n'ont pas contesté } \\
\text { juridiquement la loi 170 } \\
\text { (2/14) }\end{array}$ & 60,427 & $39 \%$ & $36 \%$ & $22 \%$ \\
\hline $\begin{array}{l}\text { Villes à statut francophone } \\
\text { qui ont contesté } \\
\text { juridiquement la loi 170 } \\
\text { (4/13) }\end{array}$ & 86,366 & $68 \%$ & $19 \%$ & $11 \%$ \\
\hline $\begin{array}{l}\text { Villes à statut francophone } \\
\text { qui n'ont pas contesté } \\
\text { juridiquement la loi 170 } \\
\text { (9/13) }\end{array}$ & $1,433,226$ & $56 \%$ & $15 \%$ & $27 \%$ \\
\hline $\begin{array}{l}\text { Incluant la Ville de Montréal } \\
\text { pré-fusion }\end{array}$ & $(1,029,828)$ & $(58 \%)$ & $(11 \%)$ & $(30 \%)$ \\
\hline $\begin{array}{l}\text { Montréal post-fusion "Une } \\
\text { île, une ville", janvier 2002 }\end{array}$ & $1,798,146$ & $46 \%$ & $31 \%$ & $20 \%$ \\
\hline
\end{tabular}
1996.

Population et langue maternelle selon le recensement de Statistiques Canada,

Ainsi, des événements politiques tels que le référendum sur l'indépendance du Québec et la polarisation ethnique qui s'ensuivit ont eu indirectement pour effet de réduire le contrôle institutionnel dont jouissait auparavant la minorité anglophone. Les services bilingues des anciennes villes à statut bilingue de l'île de Montréal sont désormais soumis au bon vouloir de fonctionnaires qui s'adonnent à être bilingues puisque ce genre de service est considéré «non-essentiel» par la loi 170 et les conventions collectives de la nouvelle ville officiellement déclarée francophone. Les services bilingues des arrondissements de quartier dépendent des enveloppes budgétaires octroyées par le conseil exécutif de la nouvelle ville de Montréal dont 
la majorité des élus sont francophones. La vitalité linguistique des communautés anglophones et allophones de Montréal se trouve donc fragilisée à moyen et à long terme.

Grâce à l'adoption des lois 170 et 171 , le Gouvernement du Partí québécois a réussi à consolider officiellement le statut unilingue français de Montréal. Ces deux lois servent à parachever les efforts de francisation de la loi 101 au niveau des municipalités. Certains stratèges de la cause francophone estiment quand même que «Nous perdons Montréal». Selon cet argumentaire, si les citoyens de souche québécoise francophone deviennent minoritaires sur l'île de Montréal en raison de l'immigration non francophone, comment assurer le statut linguistique de la seule grande métropole francophone de l'Amérique du Nord? Toujours selon cet argumentaire, comment s'assurer de l'assimilation linguistique des immigrants et comment s'assurer que les Québécois francophones demeurent majoritaires à Montréal? Force est de constater que l'étalement urbain de Montréal s'est poursuivi sans relâche au cours des vingt dernières années. Dans sa récente analyse, Marc Lévine (2002) note que l'île de Montréal a connu une perte nette par migration de 112000 francophones vers les banlieues des couronnes nord et sud du grand Montréal, entre 1986 et 1996. Ainsi, la proportion de citadins parlant le français au foyer sur l'île de Montréal est passée de $61 \%$ en 1986 à $55 \%$ en 1996, tandis que le pourcentage de citoyens s'exprimant dans une langue autre que le français à la maison passait de $39 \%$ en 1986 à $44 \%$ en 1996. Marc Lévine dresse le portrait suivant des enjeux démolinguistiques du français à Montréal :

«L'étalement urbain à Montréal est essentiellement un phénomène francophone : $80 \%$ des résidents de l'ile qui se sont installés dans les banlieues des couronnes depuis le début des années 1980 parlent le français à la maison... Non seulement les francophones ont-ils quitté l'île, mais les francophones en provenance des autres régions du Québec préfêrent s'installer dans les communautés des couronnes plutôt que dans celles de l'île ...Bref, en évaluant l'importance des tendances démolinguistiques récentes en regard de l'avenir du français à Montréal, il faut identifier très précisément les causes du déclin démographique des francophones sur l'île de Montréal depuis 1986. Le phénomène n'est pas une conséquence, comme beaucoup le craignaient dans les années 1970, d'une anglicisation massive et rapide des immigrants. Au contraire, la baisse de la proportion des francophones par rapport à la population de l'île est le résultat d'une allophonisation de l'île associée à une forte augmentation de l'immigration internationale et à un exode continuel des francophones vers les banlieues en couronne. Comme nous le verrons plus tard, cette "allophonisation" représente une tendance qui existe dans presque toutes les grandes métropoles au Canada et aux Etats-Unis» (p. 169-171)

Étant donné le nombre d'immigrants allophones de tous les horizons linguistiques, Montréal est devenue une ville multilingue et multiculturelle. Or, le gouvernement du Québec n'a pas réussi à contrer la tendance des immigrants à s'établir sur l'île de Montréal. En effet, les immigrants allophones ne se sont pas installés dans les régions du Québec où la majorité d'accueil francophone est perçue 
comme plus susceptible de faciliter leur assimilation linguistique. D'ailleurs, comment convaincre les immigrants de s'établir dans les régions du Québec délaissées par les Québécois eux-mêmes en raison du manque d'emploi et de débouchés économiques? Conscient de ses échecs, le gouvernement du Parti québécois a cherché à atteindre ses objectifs linguistiques et identitaires en réduisant le soutien institutionnel des minorités anglophones de l'île de Montréal. Si ces politiques incitent un certain nombre d'allophones et surtout d'anglophones à émigrer vers les autres provinces canadiennes, certains diront que le gouvernement du Québec sera parvenu non seulement à réduire la proportion des non francophones sur l'île de Montréal mais en même temps à réduire le risque de la partition et $\mathrm{du}$ vote anti-souverainiste lors $\mathrm{du}$ prochain référendum sur l'indépendance du Québec. En revanche, d'autres soutiendront que la fusion forcée des villes anglophones de l'ouest de l'île de Montréal constitue une menace pour le contrôle de la seule grande métropole francophone de l'Amérique du Nord. Les élus majoritairement francophones du gouvernement municipal de l'ancienne ville de Montréal doivent maintenant partager le pouvoir avec l'ensemble des élus de l'ouest de l'île de Montréal qui sont majoritairement anglophones et allophones. Ces élus ont entre autres pour mandat de représenter les intérêts linguistiques, culturels et économiques des communautés anglophones et allophones des arrondissements de l'ouest de la nouvelle ville de Montréal. Les compromis intercommunautaires nécessaires au bon fonctionnement de la nouvelle ville aideront-ils à enchâsser le statut de Montréal, plus grande ville majoritairement francophone du continent, tout en respectant les droits des minorités linguistiques anglophones et allophones?

\section{C) La loi 101 et l'éducation}

Contrairement à la plupart des autres minorités linguistiques en Amérique du Nord, les anglophones du Québec bénéficient des services d'un système éducatif de langue anglaise subventionné par l'État, de la maternelle jusqu'au niveau collégial et universitaire y compris les études graduées et post-doctorales. Ainsi, trois universités anglophones subventionnées par des fonds publics accueillent un total de près de 60000 étudiants à temps complet ou à temps partiel au premier cycle (le nombre d'étudiants inscrits dans les sept universités francophones du Québec est de 160000 au premier cycle). La majorité des anglophones inscrivent leurs enfants dans le réseau scolaire primaire et secondaire anglophone. Cependant, à l'intérieur du système anglophone, les parents anglophones ont manifesté le désir d'inscrire leurs enfants dans des programmes d'immersion en français : la proportion d'élèves anglophones dans les classes d'immersion en français est passée de $24 \%$ en 1981 à $32 \%$ en 1998. En outre, un nombre croissant d'élèves ayant l'anglais comme langue maternelle (anglophones et immigrants) fréquentent le réseau scolaire francophone : $10 \%$ en 1972 et $17 \%$ en 1995 (Québec, 1996).

Suite à la promulgation de la loi 101, les anglophones s'inquiétèrent des conséquences démographiques défavorables pour leur groupe du fait que la plupart des futurs immigrants au Québec seraient obligés d'envoyer leurs enfants dans des écoles primaires et secondaires francophones plutôt qu'anglophones (Mallea, 1984). La loi 101 a eu l'effet voulu sur les inscriptions au système scolaire primaire et 
secondaire anglophone dans la province. En effet, les effectifs allophones dans le système scolaire anglophone sont passés de $85 \%$ en 1972 à seulement $20 \%$ en 1998, tandis que les inscriptions des allophones dans le système scolaire primaire et secondaire francophone sont passés de $15 \%$ en 1972 à $80 \%$ en 1998. En conséquence, les anglophones ne peuvent plus compter sur les immigrants pour maintenir la base démographique de leur système scolaire dans la province qui comptait 231815 élèves en 1971 et seulement 98116 en 1998 (MEQ, 1999). Les études suggèrent que cette baisse du nombre d'élèves fréquentant le système scolaire anglophone découle en grande partie de la loi 101, de la conjoncture sociopolitique et économique québécoise réduisant le nombre d'Anglo-Canadiens s'établissant au Québec et du déclin de la fécondité des anglophones (Québec, 1996a). La migration de beaucoup d'anglophones unilingues vers les autres provinces canadiennes conjuguée à leur fréquentation des écoles françaises et à la popularité croissante des classes d'immersion en français contribua à l'essor du bilinguisme français-anglais chez les anglophones du Québec.

Comme dans le passé, le recensement de 1996 montre que la proportion de diplômés de l'université est plus élevée chez les anglophones du Québec (21\%) que chez les francophones (14\%) ou que dans l'ensemble de la population canadienne (16\%). Parmi ceux qui poursuivent une éducation post-secondaire, plus de $92 \%$ des anglophones du Québec ont choisi des collèges et universités anglophones, tendance qui s'est maintenue dans les années 1990 . Les effectifs des anglophones dans les CEGEP francophones ont augmenté de façon marginale, passant de $5 \%$ en 1980 à $6,6 \%$ en 1990, tandis que dans les CEGEP francophones, les inscriptions des anglophones sont restées stables, à savoir aux alentours de $7 \%$ jusque dans les années 1990. Un exode des diplômés d'université anglophones a aussi eu lieu depuis l'adoption de la loi 101. De 1976 à 1986, le taux de migration net de cette catégorie d'étudiants a atteint les $40 \%$ (26550 diplômés). Parmi les facteurs relevés dans ces études pour expliquer ce phénomène, citons l'insuffisance de la compétence en français, l'incertitude politique de la province due au projet indépendantiste et les débouchés économiques plus intéressants dans le Canada anglais. Cependant, une petite proportion de ceux qui sont partis durant cette période étaient en réalité des Anglo-Canadiens retournant dans leur province d'origine après avoir fini leurs études universitaires au Québec. La migration vers les provinces anglophones s'est maintenue dans la décennie 1986-1996 (28\% de perte totale : 21688 diplômés) et est imputable à des perspectives d'emploi plutôt sombres dues au déclin de l'économie québécoise durant cette décennie. Dans l'ensemble, durant ces 20 dernières années, parmi les diplômés d'université, les anglophones étaient douze fois plus enclins à quitter la province que les francophones. Le départ des jeunes universitaires anglophones n'a pas seulement eu un impact négatif sur le développement économique de la société québécoise dans son ensemble mais a aussi compromis la capacité de la minorité anglophone de maintenir sa vitalité démographique et institutionnelle dans la province.

La loi 101 n'a pas seulement pour effet d'augmenter la proportion des groupes allophones inscrits dans le système scolaire primaire et secondaire francophone mais a aussi influencé l'utilisation du français par les élèves allophones dans la cour de l'école et à la maison. D'après une enquête effectuée auprès d'élèves allophones inscrits dans des écoles secondaires de Montréal, dans les corridors et dans les cours de récréation, les allophones disaient utiliser surtout le français (57\% du temps), 
puis l'anglais ( $25 \%$ du temps) et, dans une moindre mesure, les langues d'origine (18\% du temps) Dans le cadre familial, avec leur fratrie, les élèves allophones déclaraient utiliser autant le français $(40 \%)$ que leur langue d'origine $(40 \%)$ tandis que l'anglais s'utilisait $20 \%$ du temps. (Giroux, 1992). Ces résultats furent confirmés par une vaste enquête effectuée auprès d'allophones inscrits dans vingt écoles francophones au niveau primaire et secondaire de Montréal (McAndrew, Veltman, Lemire, et Rossell, 2001).L'étude consistait à observer systématiquement le comportement langagier des élèves dans leurs échanges informels dans les corridors et les cours de récréation des écoles à moyenne et forte densité d'allophones. L'usage du français chez les allophones prédomine dans l'ensemble des échanges informels entre élèves dans $60 \%$ à $100 \%$ des cas et ce, dans toutes les écoles. L'usage des langues d'origine est limité aux communications intragroupe (entre allophones), ce qui était prévisible. L'utilisation de !'anglais pour les échanges informels n'est fréquente que dans deux écoles caractérisées par la présence d'une clientẻle anglophone de vieille souche. Finalement, les entrevues effectuées dans les écoles secondaires montrent que la valorisation du français «langue commune» et l'importance de son usage en milieu scolaire font l'objet d'un large consensus (McAndrew, 2002).

Une autre mesure révélatrice des préférences linguistiques des allophones consiste à examiner les inscriptions au niveau collégial et à l'université où la liberté du choix de la langue demeure intacte pour tous les citoyens de la province. L'effectif des allophones dans les CEGEP francophones est passé de $18 \%$ en 1980 à $40 \%$ en 1989 et à $46 \%$ en 1994 . Les statistiques de 1989 sont importantes dans la mesure où ce groupe représente la première génération d'étudiants allophones dont l'entrée en première année du primaire coöncidait avec la première année d'application de la loi 101 en 1978. Après l'année 1989, les chiffres reflètent le fait que beaucoup d'allophones scolarisés en français au niveau secondaire ont décidé de poursuivre leurs études collégiales dans le système francophone. En réalité, parmi les allophones scolarisés en français au niveau secondaire, une proportion de $64 \%$ se sont inscrits dans un CEGEP francophone en 1994 ; ceux qui ont opéré un transfert dans un CEGEP anglophone (36\% d'entre eux) ont pris le risque de poursuivre leurs études dans une langue autre que celle utilisée au niveau secondaire. Parmi les allophones scolarisés dans le système secondaire anglophone, très peu se sont tournés vers le français au niveau collégial (moins de1\%). Enfin, la proportion d'allophones qui ont opté pour une université de langue française est passée de $42 \%$ en 1986 à $47 \%$ en 1994 tandis que la majorité d'entre eux accordaient la préférence à une université de langue anglaise.

Comme nous l'avons déjà mentionné, bien des allophones et des immigrants établis au Québec ont adopté le trilinguisme comme stratégie d'intégration face à la coexistence et à la rivalité des deux communautés d'accueil. Les allophones et leur progéniture maintiennent la connaissance de leur langue d'origine parce que c'est leur langue socio-affective et la langue maternelle de leurs parents. Ils apprennent le français en raison de la contrainte imposée par la loi 101 dans la seule juridiction à majorité francophone en Amérique du Nord. Quant à l'anglais, ils l'adoptent parce que cette langue demeure la lingua franca de la mobilité économique et sociale au Canada et aux États-Unis (Bourhis, 1994b). Les clauses de la loi 101 avaient pour but de garantir l'apprentissage du français par les allophones et les immigrants dans le cadre scolaire. Néanmoins, la loi ne force pas les allophones à s'assimiler 
linguistiquement à la majorité francophone. Il n'en reste pas moins que certains activistes francophones comptaient sur la loi 101 pour créer un effet d'entraînement indirect, incitant à un transfert au français ainsi qu'à l'identification au milieu culturel québécois francophone de préférence au réseau culturel allophone ou anglophone. Lorsque de telles attentes ne se réalisent pas vraiment, les militants nationalistes francophones réagissent en manifestant leur impatience, voire leur intolérance à l'égard des allophones qui continuent à s'identifier à leur endogroupe, qui maintiennent leur langue d'origine au foyer ou qui préfèrent s'intégrer à la communauté d'accueil anglophone plutôt que francophone. Par ailleurs, d'autres estiment que les allophones et les immigrants n'ont aucune obligation de s'identifier uniquement en tant que francophones ou anglophones puisque l'identification multiple y compris à son groupe d'origine constitue une option possible surtout à Montréal, ville multilingue et multiculturelle dans les faits.

Étant donné que la loi 101 obligeait tous les parents francophones à inscrire leurs enfants au système scolaire public d'expression française, le pourcentage des élèves de langue maternelle française dans les écoles primaires et secondaires d'expression française est resté stable dans les vingt dernières années : $98 \%$ en 1972, $99 \%$ en 1995. Ainsi, malgré le déclin démographique de la population francophone, le système scolaire francophone a réussi à maintenir sa clientèle qui est passée de 1178133 en 1971 à 1021448 en 1998 (MEQ). Au niveau collégial et universitaire, les données gouvernementales des quinze dernières années montrent que $95 \%$ des francophones choisissent de poursuivre leurs études post-secondaires dans des institutions de langue française et $5 \%$ seulement dans des institutions de langue anglaise. Ces tendances indiquent que la loi 101 a atteint ses objectifs dans la mesure où les clauses destinées à restreindre le choix de la langue au niveau primaire et secondaire ont pour effet de maintenir les francophones au sein du système francophone au niveau collégial et universitaire. Les restrictions imposées à la liberté de choix individuelle de la majorité francophone au niveau primaire et secondaire contribuent à consolider le statut collectif du français en tant que langue de l'enseignement supérieur dans le domaine scientifique, économique et culturel, limitant ainsi la menace due à la coexistence de l'anglais dans la province.

\section{D) La loi 101 et la langue de travail}

La loi 101 avait pour objectif d'améliorer l'utilisation du français comme langue du travail dans les industries, les entreprises et les institutions financières du secteur privé à travers le Québec. La mesure la plus importante pour consolider l'utilisation du français dans le secteur privé fut l'obligation pour les entreprises de plus de 50 employés d'obtenir des certificats de francisation prouvant qu'elles se conformaient aux règlements linguistiques imposant l'utilisation généralisée du français. La plupart des entreprises se sont soumises aux obligations de francisation et la proportion des entreprises du Québec qui ont reçu leur certificat de francisation est passée de 7,7\% en 1980 à $71,6 \%$ en 1999 (Daoust, 1984 ; Maurais, 1987 ; OLF, 1998). Une étude effectuée par l'Office de la langue française (OLF) a montré que la probabilité d'utilisation du français comme langue de travail (écrite et parlée) était plus élevée dans les entreprises dotées d'un certificat de francisation (plus de 
$80 \%$ d'utilisation du français) que dans celles qui n'avaient pas de certificat (65\% d'utilisation du français ou moins ; Bouchard, 1998 ; 2002).

Durant la première décennie d'application de la loi de francisation des entreprises, un certain nombre de grandes firmes anglophones ont déplacé de façon plus ou moins évidente leurs quartiers généraux du Québec vers d'autres régions du Canada (Miller, 1984). Grâce à l'analyse des registres concernant les certifícats de francisation, l'OLF a pu montrer que les entreprises possédées par des anglophones étaient plus lentes à obtenir leur certificat de francisation que celles aux mains des francophones et des allophones (Bouchard, $1991 ; 2002)$. Le seuil atteint au début des années 1990 dans le nombre d'entreprises ayant obtenu leur certificat de francisation s'explique par des facteurs d'ordre industriel et structurel qui échappent au contrôle de l'OLF. Les firmes dont la francisation demeure problématique présentent certaines caractéristiques ; il s'agit de multinationales ayant des filiales partout dans le monde et dont la culture d'entreprise et la gestion informatique sont essentiellement anglophones ou encore d'entreprises locales à la pointe de la technologie et dont le marché est principalement international plutôt que local (aéronautique, informatique et biotechnologie ; Bouchard, 1998). Étant donné le grand rayonnement et le prestige de ces industries créatrices d'emplois, l'OLF a fait preuve de patience dans son désir de mettre sur pied des programmes de francisation dans ces entreprises (Bouchard, 2002).

L'élection de gouvernements souverainistes, les deux référendums sur la séparation du Québec, les politiques físcales et la francisation du monde du travail ont contribué au départ de beaucoup d'entreprises anglo-canadiennes. L'exode des employés et des administrateurs anglophones a eu des retentissements sur le statut des francophones et des anglophones dans le monde du travail. Par exemple, dans la région de Montréal, la proportion de gestionnaires et de cadres francophones est passée de $55 \%$ en 1971 à $68 \%$ en 1991, tandis que la proportion de cadres anglophones a décliné, passant de $34 \%$ en 1971 à seulement $18 \%$ en 1991. De même, la proportion de francophones occupant des postes administratifs supérieurs a augmenté : elle est passée de $41 \%$ en 1971 à $67 \%$ en 1991 tandis que la proportion d'anglophones occupant des postes équivalents a baissé, passant de 47 $\%$ en 1971 à $20 \%$ en 1991. Néanmoins, les réseaux linguistiques des deux solitudes du Québec perdurent dans le monde corporatif de la province. Des études ont montré que les cadres supérieurs francophones restaient limités aux firmes aux mains des francophones $(91 \%$ en $1976 ; 87 \%$ en 1993) de préférence à celles sous gestion anglophone ( $9 \%$ en $1976 ; 15 \%$ en 1993 ; Québec, 1996a).

La modernisation de la société québécoise conjuguée aux effets de la loi 101 peut aussi être à l'origine de l'amélioration des revenus des francophones par rapport à ceux des anglophones de la province. En contrôlant le niveau d'études, l'expérience et l'âge, les études ont montré qu'en 1970, les anglophones unilingues ou bilingues gagnaient $8 \%$ de plus annuellement que les francophones bilingues et $16 \%$ de plus que les francophones unilingues. En 1990, l'écart de revenus entre anglophones et francophones était considérablement réduit ou même inversé dans certains cas. Des études contrôlant différents facteurs ont montré que les anglophones unilingues et bilingues gagnaient seulement $3 \%$ de plus que les francophones unilingues en 1990 tandis que les francophones bilingues gagnaient 4 $\%$ de plus que les angíophones unilingues ou bilingues (Québcc, 1996a). D'après les recensements, la prime salariale accordée pour la connaissance de l'anglais à 
Montréal était de $16 \%$ en 1970 ; en 1980, cette prime était tombée à $6 \%$ et n'était plus que de $3 \%$ en 1990 . Vu les acquis au profit des francophones dans le monde du travail, la commission du gouvernement du Québec chargée d'évaluer la position du français depuis la loi 101 aboutit à la conclusion suivante :

«On voit que la situation dans laquelle se trouvaient les travailleurs francophones au début des années 1970, qui avait amené les auteurs de l'énoncé de politique de 1977 (loi 101) à en faire une "question de justice sociale» a été largement corrigée, ce qui peut être attribué aussi à d'autres facteurs que la Charte. Les disparités salariales défavorables aux francophones ont été réduites de $16 \%$ à 3 $\%$. Les travailleurs de langue maternelle française occupent de plus en plus leur place sur le marché du travail. Et on ne peut plus prétendre que celui-ci "est structuré de sorte que le français domine au bas de l'échelle, que le bilinguisme s'impose au palier moyen et que l'anglais domine au faîte de l'échelle») Québec 1996a, p. 70-71.

Des recherches effectuées par le CLF ont aussi montré que la loi 101 avait amélioré le statut et l'utilisation du français langue de travail dans les entreprises et les commerces (Béland, 1991). Dans la région de Montréal, des enquêtes ont montré que la proportion d'employés francophones qui déclaraient travailler le plus souvent en français (90\% du temps ou plus) était passée de $52 \%$ en 1971 à $63 \%$ en 1989, tandis que parmi les allophones, l'accroissement était plus modeste allant de $17 \%$ en 1971 à $24 \%$ en 1989 (Québec, 1996a). Les travailleurs angiophones furent le moins touchés par les mesures concernant la langue de travail puisque leur utilisation régulière du français passa de $2 \%$ en 1971 à $8 \%$ en 1989. Cependant, une baisse de l'utilisation habituelle de l'anglais au travail ( $51 \%$ du temps ou plus) fut enregistrée pour les trois groupes de travailleurs suite à la loi 101: pour les francophones, l'utilisation de l'anglais a baissé de $6 \%$ entre 1971 et 1989 alors que pour les allophones, l'utilisation de l'anglais est passée de $58 \%$ en 1971 à $37 \%$ en 1989. Cependant, les anglophones travaillaient encore le plus souvent dans leur langue malgré une baisse importante entre $1971(86 \%)$ et $1989(55 \%)$. Ainsi, les mesures de francisation de la loi 101 eurent l'effet voulu d'accroître l'utilisation du français au travail, surtout pour les employés francophones.

L'utilisation du français comme langue de travail et la proportion de Québécois francophones dans l'administration civile du gouvernement du Québec ont longtemps prévalu de sorte que le gouvernement provincial a dû mettre sur pied des campagnes de recrutement pour assurer une représentation plus équitable des anglophones et des allophones dans l'ensemble de la population. Malgré ces mesures, le gouvernement provincial a connu des lenteurs dans le recrutement des anglophones et des allophones au sein de l'administration civile, retard mentionné dans le rapport annuel de la Commission des droits de la personne du Québec de 1998 (CDP, 1998). Même si les anglophones et les allophones constituaient $18 \%$ de la population québécoise en 1996 (Statistique Canada), les représentants de ces deux communautés dans l'administration civile du gouvernement du Québec ne formaient que $3.5 \%$ des effectifs des fonctionnaires. Les analyses de la Commission des droits de la personne du Québec ont montré que cet écart ne pouvait s'expliquer par une maitrise insuffisante du français ni par un manque de compétence de la part des candidats anglophones et allophones (CDP, 1998 ; Bourhis, 2002). Beaucoup d'anglophones et d'allophones considèrent cet écart comme symptomatique de la fermeture de la société civile québécoise. Gretta 
Chambers ex-chancelière de l'université McGill résume dans ces termes le sentiment de rejet éprouvé par les anglophones:

"Le choc de la Loi 101 est désormais passé. Mais ce qu'il faut dire et redire, c'est que le statu quo, quel qu'il soit, est d'une extrême fragilité. Cela ne s'applique pas à la vie quotidienne où anglophones et francophones vivent, travaillent, commercent, voyagent et jouent côte à côte. Il se peut que les relations linguistiques ne soient pas parfaites, mais elles n'ont jamais été aussi décontractées ni aussi naturelles. Cela est dans une large mesure attribuable à la bilinguisation exceptionnellement rapide des anglophones, surtout des jeunes...C'est au niveau du discours public que les choses se gâtent. Dans les médias, dans les relations entre établissements, souvent dans l'embauche, dans le financement des entreprises et dans les secteurs où être anglophone est un désavantage, voire une tare, beaucoup d'anglophones se sentent dépaysés. Alors beaucoup de jeunes se demandent : "Qu'est-ce que je fais ici?" Un large segment de la population anglophone est convaincu que la Loi n'est plus une "charte de la langue française», mais qu'elle est devenue un instrument politiquement chargé visant à assurer aux Québécois de langue maternelle française les emplois généralement les mieux rémunérés. Vrai ou faux, tel est le stigmate qui se rattache à la manipulation des lois linguistique du Québec qui semblent toujours être sur des sables mouvants.» Chambers, 2002, p. 324-325.

Compte tenu des objectifs de francisation de la loi 101, les activistes francophones n'en continuent pas moins de déplorer la lenteur des progrès du français comme langue de travail chez les allophones et se montrent sceptiques face à la progression de l'utilisation du français enregistrée chez les travailleurs anglophones. Les militants exercent aussi des pressions pour étendre l'obligation des certificats de francisation aux entreprises de moins de 50 employés, option qui n'a pas été retenue dans les recommandations du Rapport de la «Commission des États généraux sur la situation et l'avenir de la langue française au Québec»» (Québec, 2001 ; Bouchard, 2002). Certes, les mesures prévues par la loi 101 pour améliorer le statut du français au travail permettent aux employés francophones de rester unilingues sans avoir à en subir les conséquences sur le plan du salaire et de la promotion. Néanmoins, étant donné l'intégration croissante de l'économie du Québec à celle du continent suite à la signature des accords de libre-échange sur le marché nord-américain (ALENA, 1991), on constate que les gestionnaires francophones, les cadres et les techniciens de haut niveau sont de plus en plus bilingues, utilisant l'anglais comme lingua franca du commerce international, de la science, de la technologie et des communications.

A ce propos, des pourparlers sont en cours pour la création d"une zone de libre-échange des Amériques (ZLEA), prévue pour 2005. Ainsi, le Conseil de la langue française en collaboration avec les ministres des Relations internationales du Québec et du Canada ont tenu leur premier «Séminaire interaméricain sur la gestion des langues» à Québec en août 2002 (Québec, 2002). Dans son discours de bienvenue, Madame Bredimas-Assimopoulos, présidente du Conseil de la langue française du Québec déclarait : 
"Nous savons que ces pressions vers l'uniformisation ne sont pas nécessairement malicieuses, ou même voulues; le pouvoir d'attraction des plus nombreux, des plus forts et des plus riches suffit la plupart du temps. Seules des garanties linguistiques claires et permanentes peuvent protéger les langues, mais aussi les cultures. Sans ces garde-fous, que seul un gouvernement est $\dot{a}$ même de créer, toute spécificité disparaît plus ou moins rapidement au profit du plus fort... Pour réussir, nous devrons tous et toutes sensibiliser nos diverses autorités gouvernementales $\dot{a}$ l'importance : 1) de contribuer au respect et à la promotion de la diversité linguistique des Amériques 2) de reconnaître le caractère officiel d'au moins quatre grandes langues des Amériques, soit l'anglais, l'espagnol, le portugais et le français dans le cadre du processus d'intégration des Amériques et des institutions interaméricaines qui en découleront.»

$\mathrm{Au}$ terme de ce séminaire, les participants issus des milieux politique, gouvernemental et universitaire des pays d'Amérique ont recommandé la création d'un forum rattaché à la Commission interaméricaine de la culture. La création de ce forum fait partie intégrante des sept résolutions adoptées par les participants. Ces résolutions portent sur les points suivants : 1) la reconnaissance du caractère officiel de l'anglais, de l'espagnol, du français et du portugais sur le plan supranational. Cette reconnaissance inclut la mise sur pied d'une structure de supervision et d'échange concernant la diversité linguistique des Amériques. 2) la reconnaissance du multilinguisme dans le fonctionnement des organismes interaméricains 3) la politique d'apprentissage des langues des Amériques rendant obligatoire l'enseignement d'au moins deux langues secondes ou étrangères dans le système scolaire. 4) les politiques linguistiques ayant trait à la reconnaissance des langues autochtones et des créoles des Amériques. 5) La normalisation et la diversité culturelle et linguistique incluant l'élaboration de normes techniques et terminologiques dans les quatre langues des Amériques. 6) L'affirmation de la diversité linguistique et la promotion de la diversité culturelle 7) Les politiques linguistiques visant à protéger les consommateurs dans les quatre langues des Amériques. Dans son discours de clôture, Madame Diane Lemieux, ministre de la Culture et des Communications du Québec précisait :

"Comme cela est le cas pour chaque langue officielle de vos États respectifs, le français au Québec est un élément essentiel de la cohésion sociale et de l'égalité des citoyens. Il est nécessaire d'assurer sa présence dans les secteurs où les seules lois de l'économie risquent de le faire reculer sinon disparaître, en particulier pour tout ce qui concerne l'information de nos concitoyens. Avec les autres grandes langues officielles, la langue française doit participer à la construction des Amériques plurielles».

\section{4) Conclusion : Perspectives et enjeux du fait français au Québec}


Des progrès remarquables ont été réalisés par la langue française au Québec grâce à des lois linguistiques telles que la Charte de la langue française. Par conséquent, même en tenant compte de la continuité de la transmission intergénérationnelle du français qui est demeurée forte, le cas du Québec constitue un succès dans l'histoire de l'aménagement linguistique puisqu'il a réussi à inverser le glissement linguistique (RLS, Fishman, 1991, 2001). Néanmoins, beaucoup de francophones se sentent encore menacés démographiquement en tant que minorité linguistique en Amérique du Nord, situation géopolitique qui ne risque guère de changer, que le Québec devienne ou non indépendant. Les anglophones, quant à eux, se sentent menacés en tant que minorité linguistique au Québec à la fois au niveau de la vitalité démographique, du statut et du contrôle institutionnel.

Selon beaucoup d'analystes, la loi 101 fut bénéfique dans la mesure où elle a désamorcé un conflit intergroupe potentiellement explosif entre la majorité francophone désavantagée économiquement et l'élite anglophone dominante (Rocher, 1992). La crise d'octobre 1970 qui amena l'armée canadienne sur le territoire du Québec pour neutraliser les activités du mouvement armé «Front de Libération du Québec»(FLQ) illustre bien la division de classes et le clivage politique existant au sein de la société québécoise de l'époque (Fournier,1998). Une politique d'aménagement linguistique telle que la loi 101 constitue un type d'intervention démocratique qui contribue à résoudre par des moyens institutionnels un conflit linguistique et social qui menaçait de déstabiliser la société québécoise et canadienne (Esman, 1987). Ainsi, les politiques linguistiques peuvent constituer des outils précieux permettant aux Etats multilingues de se moderniser de façon plus harmonieuse.

Pour les nationalistes québécois les plus militants, la loi 101 fut peut-être trop efficace dans la mesure où elle a contribué à réduire le sentiment de menace linguistique qui attisait le mouvement en faveur de l'indépendance du Québec. Les activistes francophones ne manquent pas de souligner les nombreuses clauses de la loi 101 , telle que conçue à l'origine, qui se trouvèrent annulées ou neutralisées par des jugements de la Cour Suprême du Canada basés sur la Charte des droits et libertés du Canada et du Québec (Bourhis, 1994a ; Veltman, 1998). Les militants dénoncent aussi les faiblesses de l'application de la loi 101 dans le monde du travail et de l'éducation. Par la même occasion, ils déplorent le pouvoir d'attraction de l'anglais qui perdure dans le domaine de la communication électronique et des média et ce, partout dans la province, pas uniquement à Montréal (Barbeau, 1998). Beaucoup de militants francophones considèrent l'indépendance du Québec comme la solution ultime qui permettra au futur État québécois d'adopter les lois linguistiques jugées nécessaires pour réellement enchâsser le statut du français tout en se préservant de l'ingérence du gouvernement canadien avec sa Charte des droits et libertés qui protège les droits individuels, sa loi sur les langues officielles (Fortier, 1994) et sa loi sur le multiculturalisme (Fléras et Elliott, 1992).

Conjuguées à un taux de natalité en déclin, les politiques linguistiques telles que la loi 101 eurent un effet négatif sur la vitalité du groupe anglo-québécois. Pour survivre en tant que minorité, les anglophones durent s'adapter en devenant une communauté bilingue partageant l'anglais, véritable lingua franca, avec les allophones et les communautés immigrantes. Toutefois, il n'est pas assuré que le soutien institutionnel dont jouit actuellement la communauté anglophone suffise à compenser l'érosion de sa base démographique. Rappelons que le soutien 
institutionnel de la communauté anglophone dépend en grande partie de sa vitalité démographique qui est justement en déclin. Les anglophones du Québec restent plus mobiles économiquement que les francophones et continuent à quitter la province quand ils sont en quête d'un meilleur emploi. Des enquêtes récentes suggèrent en outre que près d'un demi million d'anglophones quitteraient le Québec si le oui majoritaire l'emportait lors d'un troisième référendum sur l'Indépendance du Québec.

En revanche, les allophones du Québec sont moins susceptibles que les anglophones de quitter la province pour des raisons économiques ou professionnelles. Compte tenu de la rivalité des deux langues d'accueil qui se neutralisent, c'est au Québec que l'on trouve le taux le plus élevé de transmission intergénérationnelle des langues d'origine parmi les allophones établis au Canada. C'est surtout à Montréal que les allophones et les individus d'origine immigrante peuvent le plus facilement se construire des identités et des appartenances multiples, amalgamant leur propre culture d'origine avec deux cultures importantes du monde occidental liées à l'anglais et au français.

Les francophones comme les anglophones ne peuvent compter sur un accroissement de la natalité pour améliorer leur situation démographique respective dans la province. Par ailleurs, étant donné les difficultés économiques du Québec, ni les francophones ni les anglophones ne peuvent espérer attirer des citoyens en provenance des autres provinces canadiennes pour renforcer la force démographique de leur propre communauté linguistique. À défaut, les allophones et les immigrants internationaux demeurent la solution la plus prometteuse pour améliorer la situation linguistique des deux communautés d'accueil. À la fois les francophones et les anglophones dépendent de l'intégration linguistique des immigrants pour consolider leur situation linguistique à l'intérieur de la province. Cependant, parmi les effectifs des immigrants internationaux arrivant au Canada, la proportion s'installant au Québec a décliné et représente seulement $16 \%$ de la totalité des flux de la dernière décennie même si le Québec compte $23 \%$ de la population canadienne. Sans une augmentation substantielle de l'immigration ou des taux de fécondité, les démographes prévoient une amorce de déclin dans la population québécoise au cours des deux prochaines décennies (Castonguay, 1998,1999).

Traditionnellement, l'identité «québécoise» de souche était associée à une personne de langue maternelle française, qui avait des ancêtres français et qui s'identifiait à la culture canadienne-française. Cependant, surtout à Montréal, étant donné la francisation des élèves issus de l'immigration suite aux exigences de la loi 101 sur la langue de scolarité, on peut supposer que la connaissance du français devrait constituer une condition suffisante pour devenir "Québécois»(Breton, 1988). Mais la situation n'est pas aussi simple et le débat sur la citoyenneté au Québec se caractérise par de nombreux désaccords entourant la redéfinition de qui «devrait être» et qui "peut être» un "vrai Québécois» (Bourhis et al., 1997 ; Labelle, 1990 ; Taylor, 1992). A un pôle du débat idéologique québécois, selon les orientations pluralistes et civiques, les allophones et les individus d'origine immigrante peuvent être considérés comme "Québécois» dans la mesure où ils apprennent et utilisent le français comme «langue commune» de la vie publique (français au travail, dans les magasins...) et qu'ils s'acquittent de leurs devoirs et responsabilités de citoyens (voter ; payer ses impôts ; respecter les lois civiles et 
criminelles). Selon cette définition, les allophones et les citoyens d'origine immigrante n'ont aucune obligation de s'identifier aux aspirations politiques et culturelles des «Québécois de souche» ou d'y contribuer activement. De toute façon, les résultats du référendum sur l'indépendance du Québec de 1995 montrent que les "Québécois de souche» eux-mêmes sont divisés sur ce type de question puisque $60 \%$ d'entre eux ont voté en faveur du «oui» à l'indépendance et $40 \%$ ont voté «non», c'est-à-dire en faveur du fédéralisme canadien. Par ailleurs, les allophones et les communautés issus de l'immigration se sont acquittés de leur devoir de citoyens puisque leur taux de participation au vote référendaire dépassait $90 \%$, témoignant ainsi de leur degré d'intégration politique à leur société d'accueil. Dans un État qui se veut démocratique, la question du vote référendaire ou de l'allégeance aux partis politiques demeure une affaire de choix privé individuel et échappe à l'emprise de l'État. Sur la scène du débat linguistique, la position civique est justement célle qui est adoptée par le gouvernement du Québec dans son énoncé officiel sur le français comme langue de la «vie publique» (Québec, 1996b). Selon cette optique, les allophones, tout comme l'ensemble de la population, ne sont pas tenus d'adopter le français comme langue du foyer puisque l'État n'a pas à s'immiscer dans la vie privée des citoyens. Par ailleurs, pour les adeptes de l'idéologie pluraliste, le maintien des langues d'origine par les allophones représente un capital linguistique digne d'être subventionné par l'État et susceptible d'enrichir le dynamisme culturel et économique de la société québécoise (Fleras et Elliott, 1992).

L'autre pôle du débat sur la citoyenneté est plus frileux à l'égard de la diversité et est représenté par les idéologies assimilationniste et exclusionniste (Bourhis, 2001). Dans le camp francophone, les assimilationnistes s'attendent à ce que les allophones et les immigrants non seulement apprennent le français et s'acquittent de leurs devoirs civiques comme tout citoyen québécois mais aussi reprennent à leur compte les aspirations culturelles et politiques de la "majorité» des Québécois de souche. Ces revendications québécoises concernent l'adoption du français comme langue du foyer, la défense de la langue et de la culture françaises contre l'anglaise et le soutien à la souveraineté du Québec. De leur côté, les assimilationnistes anglophones s'attendent à ce que les allophones apprennent l'anglais, qu'ils s'identifient d'abord au fédéralisme canadien et qu'ils se joignent à la minorité anglophone pour défendre le fait anglais au Québec. Enfin, les exclusionnistes francophones caractérisent le pôle extrême du débat. A leur avis, il serait difficile d'accepter comme un "vrai Québécois» un individu d'origine immigrante qui n'a pas le français pour langue maternelle, qui n'a pas d'ancêtres québécois et qui ne souscrit pas aux aspirations culturelles et politiques des Québécois «authentiques».Aux yeux de ce type de nationaliste québécois, les allophones, les immigrants et les anglophones ont tendance à diluer le «fait français» au Québec et, en raison de leur nombre et de leur affiliation politique, réduisent les chances du Québec d'accéder au statut d État indépendant.

Les options pluraliste, civique, assimilationniste et exclusionniste relatives à la citoyenreté québécoise trouvent des partisans parmi les communautés d'accueil francophone et anglophone de la province (Bourhis, 2001). Ces façons d'envisager la citoyenneté sont plus ou moins populaires au sein de chacun des partis politiques de la province, à savoir le parti québécois (PQ), le parti libéral (PL) et le parti d'action démocratique du Québec (ADQ). L'issue des débats sur le type de 
citoyenneté qui finira par s'imposer au Québec aura des répercussions sur la place que pourront occuper les allophones, les immigrants, les anglophones et les autochtones dans la société québécoise qui demeure la seule province majoritairement francophone du Canada.

En conclusion, l'aménagement linguistique en faveur du français a bel et bien atteint ses objectifs tout en alimentant le débat sur la citoyenneté et la redéfinition de l'identité québécoise et canadienne. Quelle que soit l'issue du débat sur l'indépendance du Québec, des lois telles que la Charte de la langue française continueront à jouer un rôle crucial pour la survie à long terme de la seule société majoritairement francophone des Amériques.

\section{Références}

Allard, R. \& Landry, R. (1986). Subjective ethnolinguistic vitality viewed as a belief system. Journal of Multiligual and Multicultural Development, 7, 1-12.

Allard, R. \& Landry, R. (1994). Subjective ethnolinguistic vitality : a comparison of two measures. International Journal of the Sociology of Language, 108, 117 144.

Amiot, C. \& Bourhis, R.Y. (1999). Ethnicity and French-English Communication in Montreal. Poster presented at the 60th Convention of the Canadian Psychological Association, Halifax, Nova Scotia.

d'Anglejan, A. (1984). Language Planning in Quebec : An Historical Overview and Future Trends. In R. Bourhis (Ed). Conflict and language planning in Quebec. Clevedon, England: Multilingual Matters. 29-52.

Barbaud, P. (1998). French in Quebec. In J. Edwards (Ed.). Languages in Canada. New York : Cambridge University Press.

Béland, P. (1991). L'usage du français au travail : situation et tendances. Québec : Conseil de la Langue francaise. Montréal : Office de la langue française.

Béland, P. (1999). Le français, langue d'usage public au Québec en 1997. Québec : Conseil de la langue française.

Bouchard, P. (1991). Les enjeux de la francisation des entreprises au Québec 19771984. Montréal : Office de la langue française.

Bouchard, P. (1998). La francisation des entreprises au Québec : de la difficulté relative d'hier à la complexité de demain. Colloque du Comité scientifique du réseau Sociolinguistique et dynamique des langues de l'Agence universitaire de la Francophonie. Rabat, Maroc.

Bouchard, P. (2002). La langue du travail : Une situation qui progresse mais toujours tintée d'une certaine précarité. Revue d'aménagement linguistique. Hors série, Automne, 86-106.

Bourhis, R.Y. (1979). Language and ethnic interaction : A social psychological approach. In H. Giles and B. Saint-Jacques (Eds.) Language and Ethnic Relations. Oxford : Pergamon Press. 
Bourhis, R. (1982). Language policies and language attitudes : Le monde de la Francophonie. In E. B. Ryan and H. Giles (Eds). Attitudes towards language variation. London : Edward Amold. 34-62.

Bourhis, R. (1984a). Language Policies in Multilingual Settings. In R. Bourhis (Ed). Conflict and Language Planning in Quebec. Clevedon, England Multilingual Matters. 1-28.

Bourhis, R. Y. (1984b). Cross-Cultural Communication in Montreal : Two Field Studies since Bill 101. International Journal of the Sociology of Language 46 : 33-47.

Bourhis, R.Y. (1994a). Introduction and overview of language events in Canada. International Journal of the Sociology of Language. 105-106, 5-36.

Bourhis, R.Y. (1994b). Ethnic and Language Attitudes in Quebec. In J. Berry and J. Laponce (Eds.). Ethnicity and Culture in Canada : The Research Landscape. Toronto : University of Toronto Press, 322-360.

Bourhis, R.Y. (2001). Acculturation, language maintenance and language shift. In J. Klatter-Folmer \& P. Van Avermaet (Eds). Theories on maintenance and loss of minority languages. Munster, Germany: Waxmann Verlag. 5-37.

Bourhis, R.Y. (2002). Census data on «ethnic origin» in Canada : A selective retrospective and some current applications. In D. Lacorne \& P. Simon (Eds.) The measure and mismeasure of population. The statistical use of ethnic and racial categories in multicultal societies. Paris : INED (Institut National d'Études Démographiques) (à paraître)

Bourhis, R.Y. Giles, H. \& Rosenthal, D. (1981). Notes on the construction of a "Subjective vitality questionnaire' for ethnolinguistic groups. Journal of Multilingual and Multicultural Development, 2, 145-155.

Bourhis, R.Y. \& Landry, R. (2002). La loi 101 et l'aménagement du paysage linguistique au Québec. Revue d'aménagement linguistique. Hors série, Automne, 107-132.

Bourhis, R.Y. \& Lepicq, D. (1993). Québécois French and Language Issues in Quebec. In R. Posner and J.N. Green (Eds.). Trends in Romance Linguistics and Philology, Volume 5 : Bilingualism and Linguistic Conflict in Romance. The Hague and Berlin : Mouton de Gruyter, 345-381.

Bourhis, R.Y. \& Marshall, D. (1999). The United States and Canada. In J. Fishman (Ed.). Handbook of Language and Ethnic Identity. Oxford : Oxford University Press. 244-264.

Bourhis, R.Y., Moise, C., Perreault, S. \& Senécal, S. (1997). Towards an Interactive Acculturation Model : A social Psychological Approach. International Journal of Psychology, 32, 369-386.

Bourhis, R.Y. \& Sachdev, I. (1984). Vitality perceptions and language attitudes. Journal of Language and Social Psychology, 3, 97-126.

Breton, R. (1971). Institutional completeness of ethnic communities and personal relations of immigrants. In B.R. Blishen, F.E. Jones, K.D. Naegels and J. Porter (Eds.). Canadian Society : Sociological Perspectives. Toronto : Macmillan. 
Breton, R. (1988). From ethnic to civic nationalism : English Canada and Quebec. Ethnic and Racial Studies. 11, 85-102.

Caldwell, G. (1984). Anglo-Quebec : Demographic Realities and Options for the Future. In R.Y. Bourhis (Ed). Conflict and Language Planning in Quebec. Clevedon, England : Multilingual Matters. 205-221.

Caldwell, G. (1994). English Quebec : Demographic and cultural reproduction. In Bourhis, R. Y. (Ed). French-English language issues in Canada. International Journal of the Sociology of Language. 105-106, 153- 179.

Caldwell, G. (1998). English Quebec. In J. Edwards (ED.). Language in Canada. New York: Cambridge University Press. 177-201.

Caldwell, G. (2002). La charte de la langue française vue par les anglophones. Revue d'aménagement linguistique. Hors série, Automne, 27-36.

Caldwell, G. and Fournier, D. (1987). The Quebec question : a matter of population. Canadian Journal of Sociology $12: 16-41$

Canada, (1969). Royal Commission on Bilingualism and Biculturalism. Book III : The Work World. Ottawa : The Queen's Printer.

Castonguay, C. (1997). Évolution de l'assimilation linguistique au Québec et au Canada entre 1971 et 1991. Recherches sociographiques, 38, 469-490.

Castonguay, C. (1998). The fading Canadian duality. In J. Edwards (ED.). Language in Canada. New York : Cambridge University Press. 36-60.

Castonguay, C. (1999). Getting the facts straight on French : Reflections following the 1996, Census. Inroads : a Journal of opinion, 8, 57-76.

CDP (1998). Les programmes d'accès à l'égalité au Québec: Bilan et perspectives. Québec : Commission des droits de la personne et des droits de la jeunesse.

Chambers, G. (2000). Les relations entre anglophones et francophones. In : M. Plourde, H. Duval et P. Georgeault (Eds.). Le français au Québec : 400 ans d'histoire et de vie. Montréal, QC : Fides, Les publications du Québec. 319-325.

Daoust, D. (1984). Francization and Terminology Change in Quebec Business Firms. In R. Y. Bourhis (Ed). Conflict and Language Planning in Quebec. Clevedon, England : Multilingual Matters. 81-113.

Drapeau, L. (1998). Aboriginal languages : Current status. In J. Edwards (ED.). Language in Canada. New York : Cambridge University Press. 144-159.

Esman, M.J. (1987). Ethnic politics and economic power. Comparative Politics. 19, $395-418$.

Fishman, J. (1991). Reversing Language Shift. Clevedon, Avon, England.

Fishman, J. (1999) (Ed.). Handbook of Language and Ethnic Identity. Oxford : Oxford University Press.

Fishman, J. (2001). Why is it so hard to save a threatened language ? In : J.A. Fishman (Ed). Can threatened languages be saved? Clevedon, Avon, UK : Multilingual Matters. 1-22.

Fleras, A. \& Elliot, J.L. (1992). The Challenge of diversity : Multiculturalism in Canada. Scarborough, Ontario : Nelson Canada 
Fortier, I. (1994). Official Language Policies in Canada : A quiet revolution. International Journal of the Sociology of Language, 105-106, 69-97.

Fournier, L. (1998). FLQ : Histoire d'un mouvement clandestine. Montréal : Lanctôt Éditeur (533 p.)

Gagnon, F., M. McAndrew \& Pagé, M. (1996). Introduction. Pluralisme, citoyenneté et éducation. Paris, Montréal : L'Harmattan. 13-21.

Gauthier, C. \& Bourhis, R.Y. (1993). Les québécois et la question linguistique et plus particulièrement la langue d'affichage commercial : Sondage d'opinion auprès des québécois. Montréal : Crop inc. et Département de psychologie, UQAM.

Genesee, F. and Bourhis, R. Y. (1988). Evaluative reactions to language choice strategies: The role of sociosiructural factors. Language and Communication $\overline{8}$ :229-250.

Genesee, F. and Holobow, N. (1989). Change and stability in intergroup perceptions. Journal of language and Social Psychology, 8 : 17-38.

Giles, H., Bourhis, R.Y. \& Taylor, D. (1977). Towards a Theory of language in ethnic group relations. In H.Giles (Ed.). Language, Ethnicity and Intergroup Relations. London : Academic Press.

Giroux, L. (1992). Les adolescents montréalais et la télévision de langue francaise. Montréal : Université de Montréal.

Grand Council of the Crees.(1998). Never without consent : James Bay Crees stand against forcible inclusion into an independent Quebec. Don Mills, Ontario : ECW Press (280p).

Hammers, J. \& Hummel, K. (1994). The Francophones of Quebec : Language policies and language use. International Journal of the Sociology of Language, $105-106,127-152$.

Hammers, J. \& Hummel, K. (1998). Language in Quebec : Aboriginal and Heritage varieties. In J. Edwards (Ed.). Language in Canada. New York : Cambridge University Press. 384-399.

Harwood, J., Giles, H. \& Bourhis, R.Y. (1994). The genesis of vitality theory : Historical patterns and discoursal dimensions. International Journal of the Sociology of Language, 108, 167-206.

Labelle, M. (1990). Immigration, culture et question nationale. Cahiers de Recherche sociologique. 4, 143-151.

Lachapelle, A. \& Henripin, J. (1980). La Situation Démolinguistique au Canada. Montréal : Institut de Recherches Politiques.

Lambert, W.E., Hodgson, J., Gardner, R. and Fillenbaum, S. (1960). Evaluational reactions to spoken languages. Journal of Abnormal and Social Psychology 60 : 44-51.

Landry, R., Allard, R. \& Bourhis, R.Y. (1997). Profil sociolanguagiers de jeunes Francophones et Anglophones du Québec en fonction de la vitalité des communautés linguistiques. In G. Budach et J. Erfurt (Eds). Identité franco- 
canadienne et société civile québécoise. Leipig ; Leipziger Universitatsverlag. 123-150.

Landry, R. \& Bourhis, R.Y. (1997). Linguistic landscape and ethnolinguistic vitality : An empirical study. Journal of Language and Social Psychology, 16, 23-49.

Laporte, P. (1984). Status of Language Planning in Quebec : An Evaluation. In R.Y. Bourhis (Ed). Conflict and Language Planning in Quebec. Clevedon, England : Multilingual Matters. 53-80.

Legault, J. (1992). L'invention d'une minorité : Les anglo-Québécois. Montréal : Boréal

Levine, M. (1990). The Reconquest of Montreal : Language policy and social change in a bilingual city. Philadelphia : Temple University Press.

Levine, M. (2002). La question «démolinguistique», un quart de siècle après la Charte de la langue française. Revue d'aménagement linguistique. Hors série, Auatomne, 165-182.

Mallea, J. (1984). Minority Language Education in Quebec and Anglophone Canada. In R.Y. Bourhis (Ed). Conflict and Language Planning in Quebec. Clevedon, England : Multilingual Matters. 222-260.

Maurais, J. (1987). L'expérience québécoise d'aménagement linguistique. In J. Maurais (Ed) Politique et aménagement linguistique. Québec \& Paris : Conseil de la Langue francaise, Le Robert. 359-416.

Maurais, J. (1997) (Ed.) The indigeneous languages of Québec. Clevedon, England : Multilingual Matters.

Mc Andrew, M. (1996). Ethnicity in Quebec Schools. Pluralism and Education : Proceedings of the Conference on Pluralism and Cultural Enrichment in Education. Dublin, Ireland : University of Ulster. 65-84.

McAndrew, M. (2002). La loi 101 en milieu scolaire : impacts et résultats. Revue d'aménagement linguistique. Hors série, Automne, 69-84.

McAndrew, M., Veltman, C., Lemire, F., \& Rossell, J. (2000). Les usages linguistiques en milieu scolaire pluriethnique à Montréal : situation actuelle et déterminants institutionnels. Revue des sciences de l'éducation, 27, 105-126.

MEQ : Ministère de l'Éducation du Québec (1999). La situation linguistique dans le secteur de l'éducation en 1997-1998. Bulletin statistique de l'éducation, $\mathrm{n}^{\circ} 10$.

Miller, R. (1984). The Response of Business Firms to the Francization Process. In R.Y. Bourhis (Ed). Conflict and Language Planning in Quebec. Clevedon, England: Multilingual Matters. 114-129.

Moise, L.C. \& Bourhis, R.Y. (1994). Langage et ethnicité : Communication interculturelle èa Montréal, 1977-1991. Canadian Ethnic Studies, 26, 86-107.

OLF (1998). Office de la langue française : Rapport annuel 1997-1998. Montréal : Les publications du Québec.

Pendakur, R. (1990). Speaking in Tongues : Heritage language maintenance and transfer in Canada. Ottawa: Department of Multiculturalism and Citizenship. 
Québec (1972) (CommissionGendron). Rapport de la Commission d'enquête sur la situation de la langue française et sur les droits linguistiques au Québec. Québec : Editeur Officiel du Québec.

Québec (1996a). Le français langue commune. Rapport du comité interministériel sirir la situation de la langue française. Québec : Direction des communications, ministèere de la Culture et des communications. (319p)

Québec (1996b). Le français langue commune : Proposition de politique linguistique. Québec : Direction des communications, Ministère de la Culture et des Communications. (77 p.)

Québec (1997). L'évolution de la situation de l'affichage à Montréal de 1995 à 1997. Québec ; Conseil de la langue francaise. (http//www.clf.gouv.qc.ca).

Québec (2000) Loi 170, 171

Québec (2001). Le français, une langue pour tout le monde: Une nouvelle approche stratégique et citoyenne. Commission des États généraux sur la situation de la langue française au Québec. Québec : Gouvernement du Québec. (285p)

Québec (2002). Bulletin du conseil de la langue française, 10, 1-12.

Rocher, G. (1992). Autour de la langue : crises et débats, espoir et tremblement. In G. Daigle et G. Rocher (Eds.), Le Québec en Jeu : Comprendre les grands défits. Montréal : Les Presses de l'Université de Montéal. 423-450.

Rocher, G. (2000). La politique et la loi linguistiques du Québec en 1977. In : M. Plourde, H. Duval et P. Georgeault (Eds.). Le français au Québec : 400 ans d'histoire et de vie. Montréal, QC : Fides, Les publications du Québec. 273-284.

Ryan, E. B. and Giles, H. (Eds). (1982). Attitudes towards language variation. London: Edward Amold.

Sachdev, I. and Bourhis, R.Y. (1990). Language and Social Identification. In D.Abrams and M. Hogg (Eds.). Social Identity Theory : Constructive and Critical Advances. New York: Harvester/Wheatsheaf.

Sachdev, I. and Bourhis, R.Y. (1991). Power and Status differentials in Minority and Majority group relations. European Journal of Social Psychology, 21, 1-24.

Sachdev, I. \& Bourhis, R.Y. (1993). Ethnolinguistic vitality : Some motivational and cognitive considerations. In M.Hogg and D. Abrams (Eds.) Group motivation : A Social psychological perspective. New York : HarvesterWeatsheaf, 33-52.

Sancton, a. (2000). Merger mania : An assault on local Government. Montreal \& Kingston: McGill-Queen's University Press. (183p).

Schnobb, P. (2001). Faire-part pour marriages forcés : Le passé, le présent et l'avenir des 36 villes qui vont devenir Montréal et Longueil. Montréal, QC, Lanctôt Éditeur (255p).

Scowen, R. (1991). A different vision : The English in Quebec in the 1990s. Don Mills, Ontario: Maxwell Macmillan Canada

Stevenson, G. (1999). Community Besieged: The Anglophone Minority and the Politics of Quebec. Montreal : McGill-Queen's University Press. 
Taylor, C. (1992). The politics of recognition. In A. Gutmann (Ed.) Multiculturalism and the Politics of Recognition. Princeton, N.J. : Princeton University Press. 25-73.

Termote, P. and Gauvreau, D. (1988). La Situation Démolinguistique au Québec. Québec: Conseil de la Langue Française.

Thériault, J.Y. (2000). La langue, symbole de l'identité québécoise. In : M. Plourde, H. Duval et P. Georgeault (Eds.). Le français au Québec: 400 ans d'histoire et de vie. Montréal, QC : Fides, Les publications du Québec. 254-259.

Vallière, P. (1969). Nègres blancs d'Amérique. Paris : Maspéro.

Veltman, C. (1998). Quebec, Canada and the United States : Social reality and language rights. In T. Ricento and B. Burnaby (Eds.). Language and Politics in the United States and Canada : Myths and realities. Mahwah, N.J. : Lawrence Erlbaum Publishers. 301-315.

de Vries, J. (1994). Canada's official language communities: An overview of the current demolinguistic situation. International Journal of the Sociology of Language. 105-106, 37-68.

Wardhaugh, R. (1987). Languages in Competition. Oxford : Blackwell.

Woehrling, J. (2000). La Charte de la langue française ; les ajustements juridiques. In : M. Plourde, H. Duval et P. Georgeault (Eds.). Le français au Québec: 400 ans d'histoire et de vie. Montréal, QC : Fides, Les publications du Québec. 285291. 